\title{
LA NOUVELLE HISTOIRE AMÉRICAINE DE L'ÉDUCATION
}

\author{
par António NOVOA (*)
}

L'Histoire de l'Éducation connaît une période de transformations, à la fois théoriques et méthodologiques, un peu partout dans le monde. Les recherches, les congrès et les publications se succèdent, sur le plan national et international, faisant état du regain d'intérêt envers la discipline (1). Ce phénomène n'est pas le fruit du hasard, mais plutôt la conséquence d'une restructuration de l'espace scientifique et des systèmes nationaux d'enseignement, qui octroie une nouvelle pertinence sociale et académique au travail historique en éducation. La communauté scientifique s'investit dans ce débat munie de sentiments contradictoires, d'épanouissement et d'épuisement, qui sont le résultat des accomplissements des dernières décennies, mais aussi de l'envie d'aller au-delà des limites conceptuelles de l' « histoire sociale ».

Cet article se propose, justement, de voir comment ce processus de renouvellement est à l'oeuvre dans une communauté bien précise, la communauté américaine des historiens de l'éducation. Je ne suis pas le seul à me méfier du concept de nouveau/nouvelle, surtout quand on l'utilise pour désigner deux champs qui le supportent mal : l'histoire et l'éducation. Mais j'en fais un usage pragmatique, qui n'engage aucun pari idéologique ou théorique. Il s'agit, tout simplement, de réduire le corpus bibliographique d'après deux critères principaux : retenir uniquement les ouvrages publiés pendant le dernier quinquennat et éliminer les travaux de facture « empiriste » et « positiviste ».

Le but primaire de l'article est d'avancer une présentation raisonnée des nouvelles

$\left(^{*}\right)$ La préparation de ce texte m'a fait recourir à une série d'appuis personnels et institutionnels, qu'il est impossible de signaler dans une référence de bas de page. Mais je ne peux pas oublier le séjour comme professeur invité à l'Université de Wisconsin-Madison et l'amitié de Thomas Popkewitz. Qu'il me soit aussi permis de remercier les avis et les conseils de Barry Franklin, William Reese, Michael Apple, John Meyer, Ivor Goodson, Jürgen Herbst, Herbert Kliebard et Carl Kaestle. Finalement, je suis très reconnaissant à Pierre Caspard et aux deux collègues qui ont bien voulu faire la critique de la première version de l'article, dont les commentaires ont été fort stimulants et utiles.

(1) Signalons, à titre purement indicatif, la publication récente d'une série d'ouvrages consacrés à l'historiographie de l'éducation : Kadriya Salimova et Erwin V. Johanningmeier : Why sould we teach History of Education?, Moscow, 1993, 306 p.; Jean-Louis Guereña, Julio Ruiz Berrio et Alejandro Tiana Ferrer (Eds.): Historia de la Educación en la España contemporánea, Madrid, 1994, 394 p.; Marie-Madeleine Compère : L'Histoire de l'Éducation en Europe, Bern/Paris, 1995, 304 p.; Diane Ravitch et Maris Vinovskis (Eds.) : Learning from the Past: What History Teaches Us About School Reform, Baltimore \& London, 1995, 384 p.; Sol Cohen et Marc Depaepe (Eds.) : History of Education in the Postmodern Era (numéro spécial de Paedagogica Historica), Gent, 1996, 588 p.; Héctor Rubén Cucuzza (Ed.) : Historia de la Educación en debate, Buenos Aires, 1996, 316 p. 
tendances de l'histoire américaine de l'éducation (2). Du fait, il se veut un bilan construit à partir des travaux produits par une centaine de chercheurs, choisis parmi ceux qui ont une présence plus active dans le champ scientifique (congrès, publications,...). Le style adopté dans l'organisation du texte est celui de l'état-de-l'art, envisagé en tant qu'interprétation personnelle, et non pas en tant que portrait objectif des tendances d'évolution d'un champ scientifique. L'adoption d'un certain nombre de critères d'analyse sert à délimiter les marges de l'exercice, sans pour autant interférer dans la liberté de l'argumentation. Il va sans dire que l'étude ne se prétend pas exhaustive. L'intention est celle de suggérer une lecture compréhensive, basée sur l'identification des auteurs et des courants de recherche les plus dynamiques. Je me pose comme observateur des évolutions en cours essayant de produire un discours sensé sur l'historiographie américaine de l'éducation.

La première partie dresse le cadre de référence de l'article, à travers une réflexion qui se développe sur deux niveaux. D'abord, une analyse des débats historiographiques actuels, dans le but d'identifier les problèmes auxquels la recherche historique est confrontée et la façon dont ils font l'objet d'une élaboration conceptuelle aux États-Unis. On peut s'interroger sur la pertinence d'une telle « théorie de la théorie » dans l'économie du texte; mais je suis persuadé que ce détour peut aider à établir des filiations entre l'histoire et l'histoire de l'éducation, tout en introduisant des clefs de lecture qui seront, sans doute, utiles aux éducateurs et, éventuellement, aux historiens. Ensuite, une présentation briève de l'histoire de l'histoire de l'éducation aux États-Unis, qui situe les enjeux actuels de la discipline et leurs similitudes avec les problèmes généraux du travail historique.

La deuxième partie dessine une présentation de la « nouvelle » histoire américaine de l'éducation en six tendances principales, évoquées grâce aux déplacements suivants : des structures aux acteurs; du système aux écoles; de l'externalité à l'internalité; des idées aux discours; des faits aux politiques; du national au local/global.

La décision de limiter la réflexion aux auteurs américains est fort polémique, car elle tend à renforcer les faux-semblants de leurs discours internalistes, tout en empêchant une confrontation entre les historiographies de l'un et de l'autre côté de l'Atlantique. Mais il est impossible de tout faire, et cette option permet de tenir des propos plus clairs et mieux maîtrisés. Et pourtant, je n'ignore nullement que, s'il est vrai que les historiens européens de l'éducation ne sont pas lus aux États-Unis, il faut souligner l'importance que des auteurs tels que Michel Foucault, Pierre Bourdieu, Jacques Derrida ou Roger Chartier, parmi beaucoup d'autres, exercent auprès de la communauté scientifique américaine. La question est plus large qu'un simple « jeu d'influences », unilatérales ou réciproques, car dans des contextes socio-culturels contigus il est normal que les chercheurs se posent à

(2) Tout le long du texte, et pour des raisons d'économie formelle, j'utilise le terme américaine pour me référer, essentiellement, à la communauté états-unienne d'histoire de l'éducation. 
peu près les mêmes problèmes. C'est pourquoi on pourrait remplacer facilement « Amérique » par « Europe » à plusieurs endroits du texte.

Ce préambule explicite le mode de production de l'article et, par ce biais, il suggère des manières de le lire. Munis de cet avertissement, les lecteurs sont en conditions de juger des limites du style choisi, mais aussi de mieux comprendre qu'il en autorise différents usages. 


\section{PROBLÈMES DE L'HISTOIRE, ENJEUX DE L'HISTOIRE DE L'ÉDUCATION}

L'Histoire s'est édifiée à partir d'une définition matricielle de l'espace-temps. De la chronique à l'histoire « scientifique ", de l'histoire intellectuelle à la « nouvelle histoire », on voit se succéder des perspectives qui se différencient par la spécificité de leurs rapports à l'espace et au temps. C'est une problématique qui marque l'organisation interne du champ de l'Histoire, de même que les stratégies de légitimation externe de la discipline.

L'enquête historique s'est déplacée d'une insertion locale vers des encadrements nationaux, d'une territorialisation vers un jeu complexe d'interdépendances au niveau mondial. Elle est passée des événements (ou des suites d'événements) vers la délimitation d'époques, d'une périodisation stricte vers l'ouverture à la fluidité de temps de plus en plus longs. Il s'agit, dans un cas comme dans l'autre, d'évolutions qui marquent des changements d'échelle plutôt que des changements de nature, car la définition physique de l'espace et chronologique du temps n'a pas été mise en cause. Le travail historique resta situé à l'intérieur de frontières matérielles, qui essaient de maîtriser la finitude d'un monde spatio-temporellement resserré.

Anthony Giddens a bien souligné que ce n'est qu'avec l'avènement de la modernité que le temps et l'espace se sont universalisés et intégrés dans l'activité quotidienne de chacun : "La modernité globalise de telle manière que l'espace est séparé de l'endroit et est réintégré dans les dimensions vides du temps. (...) Une des caractéristiques principales de la période actuelle est le développement de liaisons complexes entre le global et le local, un local qui ne concerne pas uniquement une insertion régionale mais aussi les aspects intimes de nos vies personnelles » (3). C'est pourquoi l'analyse des conjonctions entre temps, espace et modernité requiert une réorientation conceptuelle et substantive dans la pensée sociale et dans la recherche : « cette redéfinition ne peut pas se limiter seulement à l'interprétation du temps et de l'espace; elle doit se rapporter directement à la conceptualisation de la modernité en tant que telle » (4).

Aujourd'hui, il faut penser le travail historique dans le cadre de cet éclatement des conceptions traditionnelles de l'espace-temps. L'espace ne se rapporte plus, fondamentalement, aux dimensions physiques, mais plutôt aux occupations multiples qui dé-localisent/re-localisent nos repères, filiations et identités; une vision fixiste de l'espace est contradictoire avec les "théories interprétatives », qui cherchent à comprendre la

(3) Anthony Giddens : «Foreword » in : Roger Friedland et Deirdre Boden (Eds.) : NowHere - Space, Time and Modernity, Berkeley, 1994, p. xii.

(4) Idem, p. xiii. 
nature subjective de la réalité et le sens qui lui est accordé par les différents acteurs. Le temps ne se définit plus par la séquence organisée des événements, mais plutôt par l'appropriation personnelle ou collective d'un ensemble de coordonnées qui nous situent vis-à-vis le devenir; le principe chronologique empêche une ouverture du travail historique vers des interrogations qui ne sont pas marquées par la rigidité des encadrements temporels.

Ces changements sont accompagnés d'une nouvelle vision de l'objet de l'Histoire. Il ne s'agit plus de faire la reconstitution du passé, mais bien plus de comprendre la façon dont ce passé fut amené jusqu'au présent, en influençant nos conceptions, nos manières de penser et nos langages. L'attention se porte sur les constructions de sens ou, autrement dit, sur l'amalgame de performances et de pratiques discursives qui définissent un savoir particulier, historiquement formé, qui consacre certaines manières d'agir, de sentir, de parler et de voir le monde. Les nouvelles perspectives de recherche historique sont centrées, non seulement sur la matérialité des faits, mais aussi sur les communautés discursives qui les interprètent et les inscrivent dans un espace-temps donné.

L'importance actuelle de la pensée historique se justifie, non pas en vertu de raisons disciplinaires, mais plutôt par le besoin d'historiciser l'ensemble de la pensée scientifique. Le retour de l'histoire n'est pas tant le fait du travail accompli à l'intérieur des frontières de la discipline, mais bien plus le résultat d'une historisation des manières de penser et d'approcher le monde physique et social. On sait que la rationalité moderne a nourri l'ambition de construire des vérités objectives et universelles, indépendantes des temps, des espaces et des interprétations; du fait, elle s'est organisée contre l'histoire, car ce savoir devenait inutile pour l'explication des faits scientifiques. Aujourd'hui, ce qu'on appelle la science postmoderne se définit, notamment, par la réconciliation entre la science et l'histoire, de telle façon que les taxonomies et divisions disciplinaires héritées du XIX ${ }^{\mathrm{e}}$ siècle tendent à être remplacées par des trangressions disciplinaires qui cherchent à bâtir un travail créatif et génératif; ainsi, toute connaissance scientifique devient imprégnée d'une dimension historique, ce qui oblige à mieux comprendre comment le passé survit dans les langages du présent.

\section{Problèmes de l'Histoire}

De l'Histoire aux histoires. Dans That Noble Dream, Peter Novick démontre qu'au centre du projet d'affirmation professionnelle des historiens américains se trouve, depuis le XIX ${ }^{\mathrm{e}}$ siècle, l'idée et l'idéal d'objectivité. Il explique que les critères de scientificité et de prestige au sein de la communauté historienne se sont organisés à partir d'une échelle d'éloignement/rapprochement face à une " vérité objective » sur le passé. Les éléments principaux de cette perspective peuvent être rapidement récapitulés : la croyance dans la possibilité de reconstituer la réalité du passé, en définissant la vérité par rapport à cette 
réalité; la séparation entre celui qui connaît et l'objet de la connaissance, entre faits et valeurs et, surtout, entre histoire et fiction; le principe que les faits historiques sont antérieurs et indépendants de l'interprétation; l'idée que tout ce qui existe dans le travail historique est « découvert », et non « construit », ce qui implique que le sens des faits reste inchangé même si les historiens suggèrent différentes interprétations (5).

Pendant longtemps les historiens se sont imaginés (et légitimés) en tant qu'empiristes à la recherche de faits objectifs qui préexisteraient à leur pensée. L'histoire comme le " grand récit du progrès » est l'idée structurante qui accompagne cette conception, selon laquelle la connaissance scientifique et historique pourrait remplacer la philosophie et la religion dans la définition des normes et des principes régulateurs de la vie moderne. Pendant les dernières années ces fondations ont été successivement mises en cause, donnant origine à une profusion et diversité de perspectives historiques. Elles se rencontrent sur le terrain commun d'un principe d'objectivité qui ne se pose pas en termes d'établissement de "vérités scientifiques ", mais plutôt en termes de construction de " discours véridiques »; et, en conséquence, elles insistent sur une conception de l'histoire en tant que l'une des formes discursives de compréhension du social, parmi beaucoup d'autres. Ainsi étant, elles partagent une vision nouvelle de la subjectivité, qui oblige à assumer que « la connaissance et les valeurs n'existent pas à l'extérieur des sens que les êtres humains construisent »(6).

Actuellement, il y a un désenchantement généralisé face à l'image traditionnelle, transcendentale, du travail intellectuel, ce qui conduit à un plus grand intérêt à l'égard des rapports entre savoir et pouvoir, cognition et contrôle, discours et politique (7). On assiste au déclin de modèles d'intelligibilité qui se sont imposés chez les historiens, notamment en ce qui concerne la fracture entre les objets et les sujets de la connaissance. Le retour du sujet ne traduit pas une approche idéaliste, mais, bien au contraire, l'affirmation de la subjectivité de la connaissance. Au lieu des structures, le regard est attiré par l'expérience et par la façon dont elle se constitue en discours. Au lieu de l'emprise de la quantité et de la série, l'effort de compréhension se porte sur les singularités et sur la façon dont elles se constituent en espace d'identité individuelle et collective.

Simultanément, on assiste au refus par les historiens des analyses causales et à la valorisation de la complexité, aussi bien au niveau conceptuel que méthodologique. Ceci

(5) Peter Novick : That Noble Dream - The "Objectivity Question" and the American Historical Profession, Cambridge, 1988, pp. 1-2.

(6) Dorothy Ross : Modernist Impulses in the Human Sciences 1870-1930, Baltimore \& London, 1994, p. 2.

(7) Robert Young : White Mythologies - Writing History and the West, London \& New York, Routledge, 1990, 232 p. 
va de pair avec l'abandon des visions modernes de progrès qui soutenaient le projet de l'Histoire et l'adoption d'approches qui essaient de rendre raison des différentes histoires coexistant dans un même espace-temps social. Dans cette fin du $\mathrm{XX}^{\mathrm{e}}$ siècle, l'enjeu de l'Histoire n'est plus de s'affirmer comme science sociale, mais plutôt de chercher à répondre à la crise des postulats de ces sciences. La reconstruction d'un sens pour le travail historique ne passe pas par un effort unitaire, mais, au contraire, par un investissement pluriel des différentes communautés interprétatives. À cet égard, trois problèmes méritent une attention spéciale : l'articulation entre théorie et histoire, le débat autour de l'écriture de l'histoire et la réflexion sur la nouvelle responsabilité sociale de l'historien.

Théorie et histoire. D'après Robert Young, le nouvel historicisme proclame la fin du clivage entre histoire et théorie, comme si l'histoire n'avait pas toujours été théorique, et vice-versa : « mais leur opposition a été une fiction critique nécessaire à nos temps » (8). En effet, la question est de moins en moins pertinente, car l'épaisseur théorique du travail de l'historien est, aujourd'hui, bien établie. Nous ne vivons plus une ère dominée par des systèmes stables de pensée et d'action, et nos épistémologies ne peuvent pas ignorer les défis posés par la dénommée "rupture postmoderne ». Le refus d'une téléologie de l'histoire nous oblige à accepter que rien ne peut être connu de façon sûre. C'est pourquoi il faut considérer l'histoire comme une épistémologie sociale qui interroge les rapports entre pouvoir et savoir à partir de leur localisation dans un espace et dans un temps précis (9).

Un bilan de la recherche historique accomplie pendant les dernières années révèle une utilisation de la théorie, non pas comme une sorte de préexplication, mais plutôt comme une source de nouvelles questions et d'élaborations conceptuelles plus poussées (10). Le scepticisme vis-à-vis les « grandes théories » ne signifie nullement un refus de la théorie, mais bien plus un renforcement de son rôle dans la réflexion historique, pourvu qu'elle soit utilisée de manière ajustée et adaptée. À ce propos, il est intéressant de voir la façon dont certains auteurs essaient de redéfinir le concept de théories de portée moyenne, dans le souci de mettre en oeuvre des instruments plus sophistiqués d'exploitation, d'analyse et d'interprétation des documents historiques.

Une fois le « grand consensus » (le « grand récit du progrès ») terminé, il n'y a plus de sens unitaire de l'Histoire. Une nouvelle utilisation de la théorie peut aider chacun à construire une identité propre en tant qu'historien : « Les historiens ne devraient plus se

(8) Robert Young : Torn Halves - Political conflict in literary and cultural history, Manchester \& New York, 1996, p. 164.

(9) Thomas Popkewitz : A Political Sociology of Educational Reform - Power/Knowledge in Teaching, Teacher Education, and Research, New York \& London, 1991, 290 p.

(10) Peter Burke : History \& Social Theory, Ithaca, 1993, 198 p. 
poser la question de savoir ce qu'est l'histoire légitime, tout en acceptant que, comme n'importe quelle autre discipline des humanités, ils ne possèdent pas un ensemble formalisé et largement consensuel de procédures de recherche et que rien de très intéressant ne pourrait advenir d'un effort pour le définir » (11).

Il faut éviter toute une série d'oppositions binaires qui renferment le débat intellectuel à l'intérieur de lui-même. Ceci est vrai pour des dichotomies telles que État/société civile ou pouvoir/liberté, mais aussi pour des couples qui ont marqué, traditionnellement, la pensée scientifique : mythe/réalité, fiction/fait, texte/contexte, théorie/histoire,... Il y avait un temps où ces oppositions étaient, peut-être, utiles. Aujourd'hui, le plus stimulant se passe aux frontières, aux espaces de trangression disciplinaire et de fertilisation entre différents champs scientifiques, ce qui exige le recours à une panoplie diversifiée d'outils théoriques et méthodologiques.

L'écriture de l'Histoire. La dissolution des limites est bien présente dans l'oeuvre de Hayden White, notamment quand il prétend que tout discours historique est un écrit poétique. Il soutient que les nouvelles tendances littéraires et linguistiques mettent en cause le décalage entre mots et choses, entre langage et réalité, qui a traditionnellement marqué la pensée de l'histoire; et pourtant, il se dresse aussi bien contre le contextualisme que contre l'impérialisme textuel, car pour lui l'objectif n'est pas celui de remplacer le contexte par le texte, ni l'interprétation sociale par la linguistique, mais d'essayer de comprendre l'interaction entre les deux. Tout en affirmant que la distinction principale entre l'histoire et la fiction tient à la forme, et non pas au contenu, il déclare que les rapports entre texte et contexte sont au centre des approches plus récentes, qui « sont porteuses de nouvelles manières de regarder les textes, de les inscrire au sein de discours et de lier les textes et les discours à leurs contextes » (12).

Plusieurs auteurs ont montré l'importance du langage dans la construction des arguments historiques. La « nouvelle » histoire culturelle, par exemple, a souligné que les mots ne sont pas uniquement un reflet (description ou représentation) de la réalité, mais qu'ils sont des instruments qui produisent et qui transforment la réalité. D'où le besoin d'envisager le langage comme un système qui se trouve au centre des débats sur l'identité (personnelle, sociale, collective), de façon à pouvoir tenir compte de la dimension littéraire de l'expérience sociale et de la structure littéraire de l'écriture historique (13).

(11) David Harlan : «Intellectual History and the Return of Literature » in : American Historical Review, 94 (3), 1989, p. 609.

(12) Hayden White : The Content of the Form - Narrative Discourse and Historical Representation, Baltimore \& London, 1987, p. 185.

(13) Lynn Hunt (Ed.) : The New Cultural History, Berkeley, 1989, 246 p. 
Les tendances historiographiques qui prônent un « retour à la littérature » sont très diverses, ce qui rend difficile leur définition; à beaucoup d'égards, il n'est pas approprié de parler d'une nouvelle école et il faut se contenter d'identifier quelques pratiques et sensibilités communes aux historiens qui partagent ce mode de travail, à savoir : l'utilisation de modèles complexes de communication; le refus d'interprétations fondées sur des significations préétablies; l'attention aux styles d'interaction (appropriation, négociation) et aux termes de différence (sexe, race); la présence forte des théories littéraires et de certaines approches déconstructionnistes; le souci dans l'organisation des arguments à partir d'une analyse fine des sources; la démonstration que le recours aux théories littéraires ne conduit pas à l'effacement de l'histoire (14).

À cet égard, il n'est pas étonnant de constater l'influence de Michel Foucault dans le monde anglophone (15), notamment quand il explique que son objet n'est pas le langage mais l'archive, c'est-à-dire l'existence accumulée des discours : " L'archéologie, telle que je l'entends, n'est parente ni de la géologie (comme analyse des sous-sols) ni de la généalogie (comme description des commencements et des suites), c'est l'analyse du discours dans sa modalité d'archive » (16). Les problèmes de l'écriture de l'histoire rencontrés par les nouveaux courants doivent être vus à cette lumière : le projet d'intégrer les apports littéraires sans dissoudre, pour autant, l'ensemble des pratiques dans les systèmes discursifs. Ils insistent sur le besoin d'articuler les textes avec leurs contextes de production et de réception, mais leurs pratiques restent historiques, c'est-à-dire basées sur la production/reproduction de sens à travers différents espaces-temps.

La responsabilité sociale de l'historien. Les historiens sont constamment confrontés à des « batailles de sens », à travers lesquelles ils essaient de rendre raison des documents du passé et de se construire une identité professionnelle. À l'heure actuelle, ils se heurtent à la perte de l'équilibre entre régulation et émancipation, initialement inscrit dans le projet de la modernité (17). Ils sont censés expliquer le surplus de régulation et le

(14) Adaptation d'un article de Laurie Nussdorfer : « Review Essays », History and Theory, 32 (1), 1993, pp. 74-83.

(15) À titre purement indicatif, je signale les ouvrages suivants : Stephen Ball (Ed.) : Foucault and Education - Disciplines and Knowledge, London \& New York, 1990, 214 p.; Andrew Barry, Thomas Osborne et Nikolas Rose (Eds.) : Foucault and Political Reason, Chicago, 1996, 278 p.; Graham Burchel, Colin Gordon et Peter Miller (Eds.) : The Foucault Effect: Studies in Governmentality, Chicago, 1991, 308 p.; Mitchell Dean : Critical and Effective Histories - Foucault's Methods and Historical Sociology, London \& New York, 1994, 238 p.; Jan Goldstein (Ed.) : Foucault and the Writing of History, Oxford, 1994, 310 p.; Gary Gutting : Michel Foucault's Archaelogy of Scientific Reason, Cambridge, 1989, 306 p.

(16) Michel Foucault : Dits et écrits 1954-1988, Paris, tome I, 1994, p. 595.

(17) Boaventura de Sousa Santos : Toward a New Common Sense, New York \& London, 1995, 614 p. 
déficit d'émancipation, abandonnant le rôle de « chroniqueurs du progrès » et se donnant les moyens d'une compréhension critique de ce déséquilibre, de façon à mieux saisir l'inachèvement du projet de la modernité.

La responsabilité de l'historien ne s'acquitte pas dans les réponses, mais surtout dans les questions. La valorisation d'une histoire des problèmes permet de situer son compromis intellectuel dans le temps et l'espace d'aujourd'hui. Sans renoncer à l' « éloge de la différence » entre le passé et le présent, ses repères sont dans la société à laquelle il appartient : « Le présent est moins une époque qu'une série de questions; et la cohérence avec laquelle il se présente devant nous - et sur la forme dont il est réinventé par une bonne partie des théories sociales - est quelque chose qui doit être travaillée et décomposée par la recherche historique, de façon à ce qu'il soit vu comme un rassemblement contingent d'éléments hétérogènes possédant, chacun d'entre-eux, ses propres conditions de possibilité » (18).

Pour devenir l'espace de formation d'une nouvelle compréhension de la réalité sociale, l'histoire doit éviter l'alternative entre la croyance aveugle dans les potentialités de l'explication scientifique et le scepticisme méfiant vis-à-vis tout effort de transformation et de changement. C'est dans l'entre-deux qu'il est possible de critiquer (théoriquement) sans démissioner (socialement). En effet, le nouveau paradigme ne sera pas uniquement scientifique (le paradigme d'une connaissance prudente), mais il doit aussi être social (le paradigme d'une vie décente); autrement dit : la science ne peut s'organiser uniquement d'après des « critères internes » et elle doit contribuer à une « amélioration de la vie » des hommes et des femmes (19).

Il n'y a pas d'analyses simples dans un monde tellement complexe. La primauté du politique (et non pas de la politique) est la conséquence inévitable d'une réflexion qui ne peut pas être parcellisée par tranches culturelles, sociales, économiques, ou autres. La théorie ne concerne pas uniquement le politique, au sens large du terme, elle est en elle-même une pratique politique. Ainsi, au lieu de la spécialisation de la connaissance, on est devant le besoin d'une compréhension globale des problèmes, non pas dans un sens unitaire ( «la bonne réponse »), mais plutôt dans la perspective d'une reconstruction de sens multiples à partir des histoires dont les différents groupes sont porteurs. Face à l'absence d'une rationalité globale, l'historien doit s'efforcer de montrer la pluralité de mini-rationalités qui organisent la vie sociale et le travail scientifique : c'est ici que se situe une autre praxis historienne.

(18) Andrew Barry, Thomas Osborne et Nikolas Rose, op. cit., p. 5.

(19) Boaventura de Sousa Santos : Um discurso sobre as ciências, Porto, 1987, 62 p. 


\section{Enjeux de l'Histoire de l'Éducation}

Les dilemmes de l'histoire de l'éducation sont, pour l'essentiel, les mêmes que ceux de l'histoire. Dans un cas comme dans l'autre, on a les mêmes interrogations, bien que localisées dans des objets différents et donnant origine à des processus de légitimation qui suivent un chemin propre. Comment s'imagine-t-on historien de l'éducation? Quelles références identitaires traversent le champ de l'histoire de l'éducation?

Le minimum qu'on doit exiger à un historien c'est qu'il soit capable de réfléchir sur l'histoire de sa discipline, d'interroger les différents sens du travail historique, de comprendre les raisons qui ont conduit à la professionnalisation de son univers académique. Le minimum qu'on doit exiger à un éducateur c'est qu'il soit capable de sentir les défis du temps présent, de penser son action dans les continuités et changements du travail pédagogique, de participer critiquement à la construction d'une école plus attentive aux réalités sociales.

L'histoire de l'éducation n'existe qu'à partir de cette double possibilité, qui réclame de nouvelles approches du travail historique et de l'action éducative : il faut, d'abord, accepter que l'objet de l'historien n'est pas le passé, mais tout ce qui, dans le passé, peut aider à répondre aux problèmes d'aujourd'hui; il faut, ensuite, rompre avec une vision « naturelle » ou « rationnelle » qui occulte l'historicité de la réflexion pédagogique, tout en empêchant de comprendre comment les discours scientifiques en éducation se sont construits en rapport avec le développement de groupes professionnels et de systèmes-experts de connaissance.

Aux États-Unis, l'histoire de l'histoire de l'éducation est bien connue (20). Plusieurs travaux ont expliqué les principaux moments de ce processus, en particulier après la publication, en 1960, du livre de Bernard Bailyn, Education in the Forming of American Society, qui est à l'origine de la dénommée « première vague révisionniste ». Sa critique majeure s'adresse aux « historiens-pédagogues », qui avaient réduit l'enquête aux contextes scolaires, en produisant une histoire de l'éducation présentiste, paroissiale et évangélique. Du fait, il définit un projet de rénovation autour de deux principes fondamentaux : l'histoire de l'éducation est partie intégrante de l'histoire totale et, par conséquent, elle doit abandonner une perspective institutionnelle étroite, de façon à intégrer l'ensemble des dimensions économiques, sociales et politiques; l'histoire de

(20) Cf. Sol Cohen : «The History of the History of American Education, 1900-1976 - The Uses of the Past », Harvard Educational Review, 46 (3), 1976, pp. 298-330; Barbara Finkelstein : « Education Historians as Mythmakers ", Review of Research in Education, 18, 1992, pp. 255-297; Joe L. Kincheloe : « Educational historiographical meta-analysis: rethinking methodology in the 1990s ", Qualitative Studies in Education, 4 (3), 1991, pp. 231-245; Harold Silver : « Essay review: Zeal as a historical process - The American view from the 1980s », History of Education, 15 (4), 1986, pp. 291-309. 
l'éducation doit adopter des méthodologies et des paradigmes scientifiques, refusant le « récit glorieux » d'un progrès continuellement assuré par l'école.

À partir de la fin des années soixante, en raison de l'influence des approches sociologiques et des courants marxistes, on voit se dresser une " deuxième vague révisionniste » (les révisionnistes radicaux), présentant comme figures de proue, entre autres, Michael Katz et Joel Spring. Dans des ouvrages tels que The Irony of Early School Reform (1968) et Education and the Rise of the Corporate State (1972) ils prolongent l'ouverture thématique et conceptuelle de l'histoire de l'éducation, en cherchant à comprendre les fonctions de contrôle et de reproduction sociale de l'éducation, les processus de mobilité sociale, la professionnalisation de la connaissance, le développement de l'école de masses et l'intervention de l'État dans l'enseignement, parmi tant d'autres thèmes. Plus tard, ils seront accusés d'avoir « retourné à l'envers » l'histoire traditionnelle de l'éducation sans éviter les pièges d'un fonctionnalisme de gauche...

Le travail de Diane Ravitch, The Revisionists Revised: A Critique of the Radical Attack on the Schools (1978), est la première objection de fond contre les révisionnistes radicaux : maintenant, ce sont eux qu'on accuse de produire une histoire présentiste, au service d'options idéologiques et de projets politiques bien précis. Pour le prouver, elle dénonce trois arguments fallacieux de ces auteurs : l'utilisation déterministe du concept de classe, c'est-à-dire l'idée qu'il suffit d'identifier l'origine sociale d'un individu pour expliquer ses comportements; la confusion entre motivations et résultats, car tous les événements sont lus à travers le prisme d'une intentionnalité sociale; la croyance que l'analyse de la structure d'une institution est la stratégie la plus adéquate pour en comprendre la fonction.

À partir des années quatre-vingt, on assiste à une diversification des encadrements conceptuels et des outils méthodologiques. Les approches sociohistoriques semblent incapables de rendre raison de la complexité des processus de changement dans un temps long, d'appréhender les permanences profondes et les points de rupture des dynamiques scolaires et éducationnelles. L'accumulation d'études et la multiplicité de thèmes étaient en train de produire plus de dispersion que de synthèse. S'imposait alors un virage de perspective, dans le sens d'un élargissement du concept de temps historique et d'une critique à l'utilisation préférentielle des approches quantitatives. L'histoire de l'éducation part à la recherche de nouveaux sens et de nouvelles cohérences, non pas pour limiter la diversité des points de vue, mais, bien au contraire, pour leur donner une base de sustentation.

Aujourd'hui, on utilise le préfixe post (post-révisionnisme, post-structuralisme, post-moderne,...) pour marquer un « après-quelque chose » qu'on a de la peine à bien saisir. Les temps actuels sont marqués par des débats et des interrogations sur le sens de la discipline du point de vue de l'enseignement et de la recherche. C'est une réflexion que les historiens de l'éducation affrontent avec un sentiment de confiance, qui s'explique par la 
consolidation d'une communauté interprétative dotée d'instruments propres de coopération et de travail (associations, congrès scientifiques, revues spécialisées,...). L'absence d'un modèle théorique consensuel et l'émergence d'un esprit scientifique qui tend à privilégier les espaces disciplinaires de frontière contribuent à l'élargissement du répertoire méthodologique de l'histoire de l'éducation, dans le cadre d'une mobilisation d'approches anthropologiques, culturelles, linguistiques, psychologiques et sociologiques.

Il y a deux aspects de ce processus qui méritent d'être soulignés. D'une part, l'ouverture vers toute une série de discours que la recherche historique en éducation n'avait pas encore été en mesure d'intégrer : l'appropriation des thèses féministes, ainsi que l'attention portée sur les « enfants avec des difficultés d'apprentissage » et sur les questions de la race, des ethnies, des religions ou des cultures locales, a conduit à envisager autrement les processus de scolarisation et le statut de la connaissance, suggérant de nouvelles interprétations sur les rapports individuels et collectifs à l'éducation. D'autre part, l'élargissement de l'enquête historique à d'autres publics et situations éducatives : il s'agit d'un effort pour retravailler la spécificité des problématiques de l'enseignement et l'éducation, sans que le regard soit exclusivement porté sur le scolaire au sens strict du terme.

La compréhension ultime de ces évolutions, et des dilemmes présents de l'histoire de l'éducation, passe par une référence aux changements à l'intérieur aussi bien de l'histoire que des sciences de l'éducation. Dans un cas comme dans l'autre, les années quatre-vingt marquent la fin d'une époque dominée par des systèmes de pensée (et d'action) relativement cohérents et stables. Malgré toute une série de résistances, on voit se dessiner clairement les influences du « paradigme de la science postmoderne » à l'intérieur du champ de l'histoire de l'éducation (21), ce qui pose la discipline devant les mêmes problèmes de la science historique en général, tels qu'ils ont été exposés dans la partie initiale de cet article.

Le passage de l'Histoire aux histoires consacre le besoin d'inscrire dans le portrait éducatif toute une série d'individus et de communautés qui en ont été exclus. Le projet politique de porter l'attention sur les " groupes sans voix » permet de valoriser une philosophie du sujet dans le cadre d'une réaffirmation de la subjectivité de la connaissance. Le travail historique ne s'imagine pas d'après un canon fixe, et prétendument neutral, d'objectivité, mais s'exprime par une pluralité de regards et de perspectives, pourvu que les critères de scientificité soient explicités et suivis rigoureusement.

L'articulation entre théorie et histoire est essentielle, étant donné l'importance des

(21) Cf. Sol Cohen et Marc Depaepe (Eds.) : History of Education in the Postmodern Era (numéro spécial de la revue Paedagogica Historica), 32 (2), 1996, 588 p. 
questions cognitives, épistémologiques et heuristiques dans l'espace de l'éducation. L'analyse historique réclame une sophistication de plus en plus poussée, car autrement on risque de travailler autour de l'acte éducatif, sans véritablement pénétrer dans le noyau des processus de connaissance et d'apprentissage. La méfiance ancienne des historiens de l'éducation vis-à-vis la théorie tend à être remplacée par le recours à des instruments conceptuels plus à même de rendre raison de la complexité des affaires éducatives.

Les nouveaux modes d'écriture de l'histoire suggèrent la transition d'une approche contextuelle vers une analyse textuelle, de façon à construire une meilleure compréhension des pratiques discursives à l'intérieur des espaces éducatifs. Il ne s'agit pas de reprendre la tradition de l'histoire des idées, mais, au contraire, de saisir l'organisation des discours dans le temps et d'identifier leur présence dans la pensée pédagogique actuelle. Du fait, il faut se poser la question de savoir comment les discours passés construisent nos propres manières d'envisager les débats pédagogiques et de parler des problèmes éducatifs.

L'aboutissement du projet de la modernité scolaire et l'élargissement de l'éducation et de la formation à l'ensemble de la population accordent une importance sans précédent au débat sur la responsabilité sociale de l'historien de l'éducation. Le regard historique peut aider à repenser les formes d'organisation des systèmes éducatifs, ainsi que la définition des connaissances et des valeurs nécessaires à l'éducation des élèves. Le besoin d'historiciser la réflexion éducative marque un tournant dans le sens de la valorisation du « politique » en tant que catégorie de référence de l'enquête historique.

L'histoire de l'éducation maintient, de nos jours, des fragilités congénitales et d'autres qui sont communes aux champs scientifiques de l'histoire et de l'éducation. Et pourtant, la discipline révèle une vitalité qui n'est pas le fruit du hasard. La deuxième partie de l'article sera consacrée à la présentation de travaux qui illustrent quelques-unes des tendances historiographiques qui me semblent plus stimulantes dans l'approche des questions éducatives. Je le ferai, tout en étant conscient que, dans la phase actuelle de transition paradigmatique, « la réflexion épistémologique est beaucoup plus avancée et sophistiquée que la pratique scientifique » (22); ou, autrement dit, qu'il y a un décalage entre la capacité de conceptualiser théoriquement la recherche et la capacité de mettre en oeuvre des études et des travaux qui lui donnent forme.

(22) Boaventura de Sousa Santos, op. cit., pp. 57-58. 


\section{L'HISTOIRE DE L'ÉDUCATION AUX ÉTATS-UNIS (1991-1996)}

La réflexion autour des nouvelles tendances historiographiques porte sur des ouvrages publiés entre 1991 et 1996 par une centaine d'auteurs qui travaillent dans des universités nord-américaines (23). Une première liste d'environ cinq cents auteurs fut dressée, à partir des entrées suivantes : consultation de trois bases de données bibliographiques (ERIC, Historical Abstracts et Social Sciences Citation Index); analyse des programmes des congrès annuels de History of Education Society et de la Division F (Histoire et Historiographie) de American Educational Research Association; et dépouillement de la revue History of Education Quarterly, notamment de la section de comptes rendus. Quelques auteurs qui ne se présentent pas comme historiens de l'éducation (par exemple, Michael Apple, John Meyer ou Thomas Popkewitz), mais dont les travaux exercent une influence certaine sur la recherche historique en éducation, ont été inclus dans cet inventaire.

La réduction de la liste initiale s'est faite à travers l'élimination des travaux marqués par les traditions "positivistes » et « empiristes » (environ 2/3 des auteurs identifiés) et la valorisation des chercheurs avec une présence plus forte dans la vie de la communauté scientifique. Le corpus final ne représente donc qu'une partie des recherches menées en histoire de l'éducation (24). Néanmoins, il est assez représentatif du travail accompli par les nouvelles tendances historiographiques pendant les années quatre-vingt-dix.

Il est nécessaire de lire avec prudence quelques-unes des traductions proposées. Souvent, les mêmes concepts ne recoupent pas les mêmes réalités culturelles et scientifiques. Prenons, comme exemple, le terme social history qui renvoie à une tradition fort différente de celle de l'histoire sociale. Par ailleurs, il faut être averti sur la façon dont certains auteurs européens ont été appropriés et relocalisés dans l'espace académique américain, en induisant une autre approche de leurs travaux et de leurs parcours intellectuels. Le Foucault américain est très différent du Foucault français. Et « Foucault » veut dire, aussi, Derrida, Barthes, Ricoeur ou Bourdieu, parmi tant d'autres.

Ces notes ont le but d'attirer l'attention sur le besoin d'une lecture avisée des six points suivants, qui cherchent à identifier des courants historiographiques qui ne sont jamais à l' « état pur ». Chaque recherche est conduite d'après une logique propre ayant

(23) En dehors des États-Unis, j'ai aussi pris en compte quelques chercheurs canadiens et, parfois, des auteurs d'autres pays dont l'action a des retombées sur la communauté américaine de l'histoire de l'éducation. À titre exceptionnel, et dans le but d'illustrer certains axes de recherche, j'ai retenu quelques travaux publiés avant 1991.

(24) Étant donné l'extension du corpus, les articles de revues et les chapitres dans des ouvrages collectifs n'ont été considérés qu'exceptionnellement. 
recours à des concepts et/ou à des instruments de différentes tendances. Il n'y a pas une lecture unique des auteurs et de leurs travaux; bien au contraire, il y a une pluralité d'approches et de réflexions qui peuvent être développées à propos de chaque ouvrage. La systématisation présentée n'a d'autre prétention que celle d'argumenter une interprétation personnelle sur la « nouvelle » histoire américaine de l'éducation.

Chaque point contient, de manière nécessairement succincte, quatre aspects : des observations théoriques et méthodologiques, des références bibliographiques, des présentations abrégées de quelques recherches et des commentaires critiques sur les travaux choisis. En ce faisant, je ne méconnais pas que le champ scientifique est « un monde social comme les autres et, comme le champ économique, il connaît des rapports de force et les luttes d'intérêts, des coalitions et des monopoles, et même des impérialismes et des nationalismes » (25). Je me suis donné les moyens pour analyser l'espace des prises de position, mais non l'espace des positions (26). Du fait, je ne tiens pas suffisamment en compte les conflits et les rapports de pouvoir qui traversent la communauté scientifique américaine. Ils sont à l'oeuvre, bien sûr, dans les prises de position manifestées dans les différents travaux. Mais il y a des clivages et des alliances qui tiennent, fondamentalement, à l'occupation d'un certain nombre de positions institutionnelles et symboliques.

Aux États-Unis, la communauté scientifique tend à s'organiser autour de « traditions de recherche » qui s'ignorent mutuellement, parfois de façon ostentatoire. Les accords passés entre les chercheurs entérinent des compromis et des solidarités, qui jouent un rôle essentiel dans la promotion et le prestige à l'intérieur d'un univers académique vaste et très compétitif. Ces complicités définissent un " jeu de possibles » qui a des conséquences sur le choix des objets d'étude, des encadrements conceptuels, des langages théoriques, des auteurs de référence et même des modes d'écriture. Les stratégies de distinction sont très fortes et se manifestent dans les références identitaires que chacun essaie de construire de soi-même (et de son groupe de filiation). L'affichage de certains labels - post, new,... - fait partie de cet effort de différenciation, qui constitue souvent, non seulement une façon d'assurer le prestige des chercheurs, mais aussi une question de survie académique. Dans une communauté aussi large, la volonté d'inventer le nouveau fait partie, maintes fois, d'un processus de reconnaissance publique, qui conduit à réduire la complexité des phénomènes en étude à une idée facilement repérable (voire même à une thèse polémique). Mais pour bien saisir ces querelles instestines, il faudrait aussi identifier les conditions institutionnelles et universitaires de la production scientifique aux États-Unis, projet qui n'est pas faisable dans le cadre de cet article.

(25) Pierre Bourdieu : «La cause de la science », Actes de la Recherche en Sciences Sociales, 106-107, 1995, p. 3.

(26) Je reprends ici les formules avancées par Pierre Bourdieu : « Le champ littéraire », Actes de la Recherche en Sciences Sociales, 89, 1991, pp. 4-46. 
Les considérations antérieures ne s'appliquent pas exclusivement à la communauté académique des États-Unis, bien qu'elles soient majorées par les règles de fonctionnement du monde scientifique et universitaire américain. Elles cherchent à relativiser la présentation des tendances nouvelles de l'histoire de l'éducation, en attirant l'attention sur le besoin de les insérer dans un champ plus large de conflits de pouvoir et de rapports au savoir.

\section{Des structures aux acteurs : Vers une histoire expérientielle et littéraire?}

L'effort de passage des systèmes globaux d'interprétation hérités de l'histoire sociale des structures vers la valorisation des singularités individuelles et collectives est en train de donner une nouvelle dimension aux travaux centrés sur les acteurs éducatifs. Le retour du sujet renforce des approches expérientielles qui tendent à remplacer une vision du monde de l'éducation comme « structure » et comme « représentation ». Les personnes retrouvent leur place dans le portrait historique de l'éducation.

Après avoir raconté l'histoire des contextes scolaires, la recherche se tourne vers les subjectivités, en essayant de rendre raison de l'expérience des individus - et des individus intégrés dans leurs communautés d'appartenance - et de montrer comment ils vivent leurs parcours éducatifs. Il devient impossible de retracer une histoire malgré les personnes et leurs projets, ce qui suppose l'émergence d'une nouvelle épistémologie du sujet. La pluralité des histoires tient compte de la conscience de chacun vis-à-vis sa propre vie, non pas uniquement dans une perspective individuelle, mais aussi en tant que sujets appartenant à des « communautés de sens ». La reconstruction des identités personnelles est au centre de ces perspectives expérientielles, qui suggèrent une nouvelle vision historique des différents acteurs éducatifs (enfants/élèves, enseignants, femmes,...).

Pendant les dernières années, plusieurs chercheurs ont produit des travaux basés sur des témoignages écrits ou oraux, des autobiographies, des journaux et d'autres documents qui permettent de regarder autrement les expériences de vie des enfants/élèves (27). Un des ouvrages les plus connus a été écrit par Harvey Graff, Conflicting Paths Growing Up in America (1995). À partir d'une collection de 500 mémoires personnelles (journaux, autobiographies, lettres,...), l'auteur approche la difficile croissance des enfants

(27) Cf. Glen H. Elder, John Modell et D. Parke Ross (Eds.) : Children in Time and Place: Developmental and Historical Insights, Cambridge, 1993, 290 p.; Harvey J. Graff : Conflicting Paths Growing Up in America, Cambridge, 1995, 426 p.; Emmy E. Werner : Pioneer Children on the Journey West, Boulder, 1995, 202 p. Cf. aussi Barbara Finkelstein : « Revealing Childhood, Adolescence, and Youth in the History of Education : Approaches in the 1990s », Paedagogica Historica, 32 (2), 1996, pp. 453-474. 
dans les tensions entre stabilité et changement, entre liberté individuelle et contrainte sociale. Les concepts de « conflit » et de « parcours » organisent une lecture historique qui valorise des moments-clef dans le passage de l'enfance à la vie adulte. Malgré l'incapacité pour rendre plus complexes les liens entre les dimensions biographiques et les structures sociales, ce qui empêche une interprétation plus fine des histoires de vie, le travail de Graff a le mérite d'attirer l'attention sur une histoire qui se veut à la première personne du singulier.

Un autre groupe d'auteurs marque une rupture conceptuelle plus profonde, traitant les mémoires des enfants/élèves comme des textes. Du fait, ils adoptent des stratégies de l'histoire littéraire, soulignant de façon plus nette la transition des approches contextuelles vers les analyses textuelles. Les travaux de Barbara Beatty sur la culture des enfants, de Barbara Finkelstein sur le gouvernement des jeunes, de Christine Griffin sur les représentations de la jeunesse ou de Theresa Richardson sur le mouvement hygiéniste et les politiques sociales illustrent bien les orientations de ce courant historiographique (28). Au fond, il s'agit de ne pas prendre les voix des acteurs comme un matériel au premier niveau, mais de les considérer comme des textes et de les soumettre à une analyse plus sophistiquée, dépassant ainsi la dichotomie traditionnelle entre les voix (« naturelles ») et les textes (« construits »).

Des évolutions semblables peuvent être signalées dans le travail historique sur les enseignants, dans le cadre d'un intérêt croissant envers l'expérience et les parcours de vie. Mettant l'accent sur les identités personnelles et professionnelles, ces courants de recherche n'occultent pas leur projet intellectuel et politique : " donner la voix » aux enseignants, qu'ils considèrent comme un groupe qui a été « réduit au silence » par le récit historique traditionnel (29). Les essais rassemblés par Richard Altenbaugh dans son ouvrage The Teacher's Voice (1992) mettent bien en exergue cette ligne de réflexion. Organisés en trois parties, qui approchent le processus de féminisation, l'intégration des

(28) Cf. Barbara Beatty : Preschool Education in America - The Culture of Young Children from the Colonial Era to Present, New Haven \& London, 1995, 252 p.; Barbara Finkelstein : Governing the young: Teacher behavior in popular primary schools in 19th-century United States, New York, 1989, 366 p.; Christine Griffin : Representations of Youth - The Study of Youth and Adolescence in Britain and America, Cambridge, 1993, 254 p.; Theresa Richardson : The Century of the Child - The Mental Hygiene Movement and Social Policy in the United States and Canada, Albany, 1989, 274 p.

(29) Richard J. Altenbaugh (Ed.) : The Teacher's Voice - A Social History of Teaching in Twentieth Century America, London, 1992, 228 p.; Marilyn M. Cohn \& Robert B. Kottkamp : Teachers - The Missing Voice in Education, Albany, 1993, 358 p.; Ivor F. Goodson (Ed.) : Studying Teachers' Lives, London, 1992, 254 p.; Ivor F. Goodson \& Andy Hargreaves (Eds.) : Teachers' Professional Lives, London, 1996, 238 p.; Jürgen Herbst : And Sadly Teach: Teacher Education and Professionalization in American Culture, Madison, 1989, 232 p.; Pamela Bolotin Joseph \& Gail E. Burnaford (Eds.) : Images of Schoolteachers in Twentieth-Century America, New York, 1994, 300 p.; Hannu Simola et Thomas Popkewitz (Eds.) : Professionalization and Education, Helsinki, 1996; Donald R. Warren (Ed.) : American Teachers: Histories of a profession at work, New York, 1989, $472 \mathrm{p}$. 
enseignants dans les différentes communautés et les débats sur la professionnalisation, les chapitres du livre cherchent à repondre à deux défis : remplacer le regard structurel de l'histoire de l'éducation par une attention privilégiée aux expériences et aux « voix » des enseignants; valoriser les histoires locales au détriment des descriptions larges des contextes éducatifs. Le recours systématique à l'histoire orale (entretiens et témoignages) permet aux auteurs d'accorder une plus grande visibilité à la présence et au rôle des enseignants dans l'arène de l'éducation. Révendicant une filiation dans la «nouvelle » histoire sociale, cet ouvrage représente une contribution historique appréciable, malgré l'approche un peu simpliste des liens entre pratique et théorie, entre voix et texte et, surtout, entre les niveaux micro et macro d'analyse des contextes éducationnels.

Et pourtant, les influences les plus décisives concernant l'histoire expérientielle et littéraire ont comme origine les études féministes, telles qu'elles se sont développées le long de la dernière décennie. Ce ne fut pas tant l'introduction du critère genre, mais plutôt l'affirmation de la subjectivité comme espace de connaissance, qui a permis à ces études de révolutionner le champ des sciences sociales et humaines. Le concept d'expérience, en tant qu'appropriation personnelle des événements vécus (ou en tant que processus de construction identitaire), est au centre d'un tournant dans le travail historique. Le langage se produit comme un élément d'affirmation individuelle et collective et devient, ainsi, essentiel au « dévoilement d'histoires inconnues ». Kathleen Canning a tout à fait raison de souligner que les historiennes féministes ont été les premières à découvrir le pouvoir des discours dans la construction des différences sexuelles et dans la consolidation d'autres distinctions dans les pratiques sociales et institutionnelles (30). Dans ce sens, la mobilisation du concept d'expérience n'est pas une stratégie pour éviter la problématique du pouvoir; bien au contraire, c'est la meilleure manière de montrer comment le langage et le silence fonctionnent dans la reconstruction des vies personnelles et professionnelles.

L'articulation de la « dimension expérientielle » et de l' « imagination littéraire » est l'un des apports les plus stimulants des perspectives féministes, comme l'on peut constater dans de nombreux travaux historiques en éducation (31). Je prends comme exemple

(30) Kathleen Canning : «Feminist History after the Linguistic Turn : Historicizing Discourse and Experience », Signs - Journal of Women in Culture and Society, 19 (2), 1994, pp. 368-404.

(31) Cf. Sari K. Biklen : School Work: Gender and the Cultural Construction of Teaching, New York, 1995, 208 p.; Kathleen Casey : I answer with my life: Life histories of women teachers working for social change, New York, 1993, 196 p.; Geraldine J. Clifford : "Equally in View" - The University of California, Its Women, and the Schools, Berkeley, 1995, 104 p.; Jennifer M. Gore : The Struggle for Pedagogies - Critical and feminist discourses as regimes of truth, New York \& London, 1993, 188 p.; Catherine Hobbs (Ed.) : Nineteenth-Century Women Learn to Write, Charlottesville, 1995, 344 p.; Carmen Luke et Jennifer Gore (Eds.) : Feminisms and Critical Pedagogy, New York \& London, 1992, 220 p.; Patrice McDermott : Politics and Scholarship: Feminist Academic Journals and the Production of Knowledge, Urbana, 1994, 198 p.; Sue Middleton : Educating Feminists - Life Histories and Pedagogy, New York \& London, 1993, 210 p.; Claudia Nelson et Lynne Vallone (Eds.) : The Girl's Own: Cultural Histories and the Anglo-American Girl, 1830-1915, Athens, 1994, 296 p.; Alison Oram : Women teachers and feminist politics, 1900-1939, Manchester \& New 
l'ouvrage de Kathleen Casey, I answer with my life: Life histories of women teachers working for social change (1993). Travaillant à partir des histoires de vie d'enseignantes appartenant à trois groupes d'activistes (soeurs catholiques radicales, juives de gauche et afro-américaines), l'auteur propose un récit historique où le critère genre interagit avec la race, l'ethnie et la religion. Ayant le souci de prendre ses narratrices comme des sujets créant leur propre histoire plutôt que comme des objets de recherche, Kathleen Casey met en relief la dimension politique des approches autobiographiques : « Dans ces histoires de vie ce ne sont pas seulement l'état et les institutions qui sont envisagés comme terrains de lutte politique; le langage et même l'identité personnelle deviennent aussi des arènes d'activité politique » (32).

En effet, le passage d'une histoire des structures vers une histoire des acteurs est un projet essentiellement politique, qui acquiert des contours bien précis aux États-Unis. L'idée d'écrire une histoire « alternative » relève de l'intention de bien montrer comment les termes de différence (le genre, mais aussi la race, la classe, l'ethnie,...) agissent dans le domaine éducatif (33). C'est une littérature qui puise ses racines dans une référence d'action, voire même de réorganisation de la vie sociale et culturelle, ce qui lui accorde des caractéristiques uniques. Il faut néanmoins reconnaître que la naïveté des premiers temps est en train de donner origine à une sophistication théorique et conceptuelle qui transforme ces approches en une source importante de renouvellement de l'histoire de l'éducation.

\section{Du système aux écoles : Vers une « nouvelle "} histoire sociale?

La « nouvelle » histoire sociale est l'une des tendances les plus difficiles à cerner, car elle n'a pas une spécificité propre et se situe à la croisée de plusieurs traditions théoriques et méthodologiques. Les auteurs présentés par la suite pourraient être

York, 1995, 258 p.; John L. Rury : Education and Women's Work: Female Schooling and the Division of Labor in Urban America, 1870-1930, Albany, 1991, 278 p. Cf. aussi Sandra Acker : "Gender and Teachers' Work », Review of Research in Education, 21, 1995-1996, pp. 99-162; Mary Leach : « Toward Writing Feminist Scholarship into History of Education », Educational Theory, 40 (4), 1990, pp. 453-461.

(32) Kathleen Casey, op. cit., p. 158.

(33) Cf. David W. Adams : Education for extinction - American indians and the boarding school experience, 1875-1928, Lawrence, 1995, 396 p.; Michael C. Coleman : American Indian Children at School, 1850-1930, Jackson, 1993, 230 p.; Elizabeth L. Ihle (Ed.) : Black Women in Higher Education: An Anthology of Essays, Studies, and Documents, New York, 1992, 342 p.; Devon A. Mihesuah : Cultivating the Rosebuds: The Education of Women at the Cherokee Female Seminary, 1851-1909, Urbana, 1993, 212 p.; Harry Morgan : Historical Perspectives on the Education of Black Children, Westport, 1995, 238 p.; Marjorie Murphy : Blackboard Unions - The AFT \& The NEA, 1900-1980, Ithaca \& London, 1990, 286 p.; Stanley W. Rothstein : Schooling the poor - A social inquiry into the American educational experience, Westport, 1994, $190 \mathrm{p}$. 
facilement intégrés dans d'autres catégories, notamment dans l'histoire expérientielle et littéraire ou dans la " nouvelle » histoire culturelle. D'autre part, le concept même d'histoire sociale - ou, de façon plus précise, de social history - recouvre fréquemment des réalités très différentes, voire antagoniques.

En 1988, Edward McClellan et William Reese ont coordonné un livre de référence, The Social History of American Education (34), basé sur un recueil d'articles publiés dans la revue History of Education Quarterly, créée en 1961. Le projet des auteurs était de donner visibilité à une génération de " talentueux » historiens de l'éducation qui, très influencés par le défi intellectuel lancé par Bernard Bailyn en 1960 et par les enjeux politiques des années soixante, ont été à l'origine d'un mouvement connu sous le nom de " nouvelle histoire sociale ». Une vision critique du développement de l'école américaine, l'application des méthodes des sciences sociales à l'enquête historique et l'utilisation d'analyses quantitatives caractérisent le mode de travail de ces auteurs qui ont obtenu des « réussites remarquables pendant le dernier quart de siècle » (35).

Qu'est'il devenu de cette génération à partir de la fin des années quatre-vingt? Voilà la question qui oriente ce point, lequel porte comme sous-titre : «Du système aux écoles ». En effet, si la rupture des années soixante s'est exprimée par l'éloignement d'une histoire monographique des institutions scolaires et par l'adoption de perspectives sociologiques d'analyse des systèmes d'enseignement, la transition des années quatre-vingt peut être illustrée par un retour aux écoles en tant qu'éléments organisationnels qui permettent de comprendre l'ensemble des problématiques éducatives (36). Dans le premier cas, on imagine qu'en connaissant le système on en connaît les écoles, tandis que, dans le second cas, on prétend qu'à travers l'étude d'une école il est possible d'entrer dans le fonctionnement du système.

L'identification de l'école en tant qu'espace pertinent de réflexion est le fruit d'un

(34) B. Edward McClellan et William J. Reese (Eds.) : The Social History of American Education, Urbana \& Chicago, 1988, 370 p.

(35) Idem, p. ix.

(36) Cf. Alan J. DeYoung : The Life and Death of a Rural American High School: Farewell Little Kanawha, New York \& London, 1995, 342 p.; John I. Goodlad, Roger Soder et Kenneth A. Sirotnik (Eds.) : Places Where Teachers Are Taught, San Francisco, 1990, 436 p.; Gerald Grant : The World We Created at Hamilton High, Cambrige \& London, 1988, 286 p.; David Labaree : The Making of an American High School: The Credentials Market and the Central High School of Philadelphia, 1838-1939, New Haven, 1988, 208 p.; Jeffrey Mirel : The Rise and Fall of an Urban School System - Detroit, 1907-1981, Ann Arbor, 1993, 456 p.; William J. Reese : The Origins of the American High School, New Haven, 1995, 326 p.; Susan F. Semel : The Dalton School - The Transformation of a Progressive School, New York, 1992, 206 p.; Kenneth Teitelbaum : Schooling for "Good Rebels" - Socialist Education for Children in the United States, 1900-1920, Philadelphia, 1993, 258 p.; Arthur Zilversmit : Changing Schools: Progressive Education Theory and Practice, 1930-1960, Chicago, 1993, $252 \mathrm{p}$. 
important changement de vision, d'abord dans les cercles sociologiques avec l'adoption d'un méso-niveau d'analyse, plus tard dans les milieux historiques, notamment avec l'emprunt des outils de l'ethnographie et de la micro-histoire. La motivation n'est pas celle de refaire l'histoire des institutions, mais, au contraire, d'utiliser les écoles comme points d'ancrage de l'histoire de l'éducation. Par conséquent, l'organisation scolaire est approchée comme le lieu social et symbolique dans lequel se jouent les projets des différents groupes sociaux et des individus, se concrétisent les processus de production et de reproduction sociale et s'organisent les pratiques d'émancipation (mobilité sociale) et de régulation (contrôle social).

$\mathrm{Au}$ lieu des enquêtes larges et des regards déterministes sur l'évolution des systèmes scolaires, ces auteurs se tournent, dorénavant, vers des analyses plus fines, localisées dans la vie et dans le fonctionnement des écoles. Sans rester prisonniers des sites institutionnels, ils développent leurs réflexions dans le sens d'éclairer les processus d'innovation scolaire, de mobilité sociale, de certification des élèves ou de passage à la vie active, parmi beaucoup d'autres. L'analyse consacre, d'habitude, une attention spéciale à l'insertion locale de l'école, aussi bien du point de vue social que politique et économique.

Il y a des orientations qui se maintiennent dans le travail de ces auteurs: d'abord, un objectif clairement assumé de contribuer à l'élucidation des débats actuels; ensuite, une référence systématique aux contextes socio-économiques des écoles; finalement, un rapport très étroit aux sources « classiques » de l'histoire de l'éducation. Mais il y a aussi des évolutions bien marquées, en ce qui concerne une prise de distance vis-à-vis les explications structuralistes, l'adoption de pratiques méthodologiques de plus en plus diversifiées et la compréhension du langage en tant qu'élément d'organisation - et pas uniquement de description - des phénomènes sociaux et éducatifs. Ces permanences et évolutions sont bien visibles dans quelques ouvrages qui seront brièvement présentés.

Le travail de David Labaree, The Making of an American High School - The Credentials Market and the Central High School of Philadelphia, 1838-1939, publié en 1988, analyse la façon dont la politique et le marché ont influencé l'histoire des écoles secondaires aux États-Unis (37). À travers l'utilisation de la métaphore du marché, l'auteur examine un large éventail de sources, procédant, à la fois, à des analyses textuelles et à des calculs statistiques assez complexes. Son argument central est que ces écoles ont été créées au XIX $\mathrm{e}^{\mathrm{e}}$ siècle pour former des citoyens pour la «nouvelle République », mais qu'elles ont vite été investies comme une voie pour obtenir un meilleur statut social (38). La valeur des certificats a joué un rôle décisif dans l'équilibre entre les deux fondements

(37) High Schools : Écoles secondaires développées à partir de 1821, qui correspondent à ce qu'il est convenu d'appeler le « secondaire supérieur », c'est-à-dire entre la $9^{\mathrm{e}} / 10^{\mathrm{e}}$ et la $12^{\mathrm{e}}$ année de scolarité.

(38) David Labaree, op. cit., p. 1. 
de ces écoles : la « vertu républicaine » et le « commerce capitaliste » (39).

Deux autres travaux suivent cet axe de recherche. Dans The Origins of the American High School (1995), William Reese se penche sur les changements sociaux et les débats politiques qui ont marqué l'origine des écoles secondaires. La recherche porte sur les écrits des éducateurs et des directeurs d'école, des revues d'étudiants, des mémoires, des biographies et des travaux d'élèves qui permettent de retracer la vie institutionnelle et de comprendre les différents enjeux individuels et collectifs. Le concept de pouvoir est mobilisé pour expliquer la mise en oeuvre par l'État des dispositifs de régulation des écoles et pour saisir la façon dont les projets réformateurs ont été reconstruits au niveau local. Dans The Life and Death of a Rural American High School (1995), Alan DeYoung raconte la naissance, le développement, le déclin et la mort d'une école rurale américaine, entre 1915 et nos jours. À travers le recours à des entretiens (histoire orale) et à des observations ethnographiques, l'auteur construit un récit à l'envers - du présent vers le passé -, où il cherche à cerner l'identité culturelle de l'école, ainsi que l'importance qu'elle a jouée dans la vie de la communauté locale : « Ceci n'est pas l'histoire d'une école qui a échoué. C'est l'histoire d'une école qui a contribué à former les ressources humaines dont l'Amérique moderne avait besoin (...) et qui a aidé à construire et à reconstruire une communauté locale, à une période où les communautés locales étaient encore des lieux importants de la vie sociale quotidienne » (40).

Une dernière référence mérite d'être faite au travail de Jeffrey Mirel, The Rise and Fall of an Urban School System (1993), qui analyse la réalité de Detroit entre 1907 et 1981. Situant son approche dans le courant post-révisionniste, l'auteur met en cause les explications historiques antérieures, qui regardaient les minorités urbaines et les communautés ouvrières comme étant essentiellement passives, c'est-à-dire comme « des victimes incomprises des changements sociaux ou éducatifs plus vastes » (41). Du fait, il construit un argument autour de l'idée des écoles en tant qu'institutions « conflictuelles » dans lesquelles les classes travailleuses, les groupes ethniques et les organisations civiques se sont affrontés avec les intérêts établis autour de la direction des politiques éducatives et des pratiques scolaires. La consultation détaillée d'archives locales et institutionnelles et l'utilisation ajustée de la théorie ont permis à Jeffrey Mirel de produire des interprétations nouvelles et originales (42).

(39) À propos de cet argument, cf. aussi David J. Hogan : « 'To Better Our Condition': Educational Credentialing and the 'Silent Compulsion of Economic Relations' in the United States, 1830 to the Present », History of Education Quarterly, 36 (3), 1996, pp. 243-270.

(40) Alan DeYoung, op. cit., p. 329.

(41) Jeffrey Mirel, op. cit., p. x.

(42) Sur le concept d'utilisation ajustée de la théorie, cf. Carl Kaestle : « Theory in educational history : a middle ground » in : Ronald Goodenow et William Marsden (Eds.) : The City and Education in Four Nations, 
Ces auteurs illustrent bien des options et des modes de travail qui sont en plein essor. Ils sont parmi les historiens de l'éducation les plus prestigiés, exerçant une forte influence sur l'ensemble de la politique scientifique (organisation de congrès, direction de revues, définition de politiques d'édition,...). C'est pourquoi il serait souhaitable qu'ils adoptent une attitude plus ouverte vis-à-vis d'autres historiographies de l'éducation, notamment européennes. Mais le sens qu'ils accordent à la recherche historique, le rapport proche aux sources, la flexibilité dans le maniement de la théorie et l'attention portée sur l'écriture leur permettent, sans doute, d'avancer des thèses intellectuellement sophistiquées sur la genèse et le développement des écoles aux États-Unis.

\section{De l'externalité à l'internalité : Vers une} «nouvelle » histoire culturelle?

L'un des axes de reconceptualisation du travail historique en éducation passe par l'adoption de points de vue qui font partie de l'agenda de la « nouvelle » histoire culturelle. Il s'agit de passer de l'analyse de l'externalité des processus éducatifs, soulignant la longue durée des changements et des continuités en éducation, à l'analyse de l'internalité du travail scolaire, notamment aux moments de conflit et de rupture. Le développement du curriculum, la construction de la connaissance scolaire, le fonctionnement des écoles, l'organisation du quotidien des élèves et des enseignants : voilà quelques-unes des problématiques que la recherche essaie d'approcher à l'aide de nouveaux outils théoriques et méthodologiques.

L'école a toujours été conçue comme un lieu de culture : d'abord, selon une acception idéalisée d'acquisition des connaissances et des normes « universelles »; plus tard, dans une perspective critique d'inculcation idéologique et de reproduction sociale. Dans un cas comme dans l'autre, peu d'attention fut accordée au travail interne de production d'une culture scolaire, qui est en rapport avec l'ensemble des cultures en interaction dans une société donnée, mais qui contient des spécificités propres qui ne peuvent pas être adressées uniquement à travers le prisme des surdéterminations du monde extérieur. Aujourd'hui, plusieurs auteurs s'efforcent de rendre raison de l'action accomplie au sein des institutions scolaires; en effet, comme reconnaît Herbert Kliebard, « beaucoup d'intérêt a été porté à la question de savoir qui fréquente l'école, mais très peu d'énergies ont été consacrées à la compréhension de ce qui se passe après que les enfants et les jeunes franchissent la porte du bâtiment scolaire » (43).

Cambridge, 1992, pp. 195-204.

(43) Herbert Kliebard : The Struggle for the American Curriculum 1893-1958, New \& London, 1986, p. $\mathrm{x}$. 
Il est facile d'identifier une série d'ouvrages et d'auteurs qui s'inscrivent dans cette ligne de pensée et qui peuvent être regroupés autour de deux grands domaines de réflexion : d'une part, les études qui ont pour objet l'histoire du curriculum et, d'autre part, les travaux qui balancent les permanences et les changements de l'acte éducatif et de l'organisation scolaire.

L'histoire du curriculum ne se centre pas essentiellement sur une approche épistémologique, mais plutôt sur la façon dont les connaissances se sont historiquement constituées, légitimées et organisées dans l'espace scolaire. L'analyse du processus de fabrication du curriculum permet d'éclairer les raisons du choix et de l'exclusion de certaines formes de connaissance, ainsi que d'en étudier les effets du point de vue personnel et social : « l'histoire du curriculum porte, de manière critique, sur ce qui est pris en tant que connaissance dans certaines périodes et lieux, et non sur l'établissement de ce qui est vrai ou valide » (44). Simultanément, cette histoire est intimement liée aux effets sociaux des choix concernant les cursus scolaires : « La question fondamentale ne s'épuise pas dans l'identification de ceux qui ont accès à l'école ou de ceux qui ne l'ont pas, mais elle exige qu'on soit capable d'expliquer comment les dispositifs sociaux sont conçus pour séparer l'accès aux différentes formes de savoir » (45).

L'approche historique du curriculum se dédouble en plusieurs objets de travail qu'il est possible d'illustrer, à titre indicatif, à travers quatre ouvrages. Dans Forging the American Curriculum (1992), Herbert Kliebard rassemble une série d'essais qui cherchent à déconstruire une vision " naturelle ", en insistant sur l'idée de curriculum en tant qu'arène où différents intérêts sociaux se confrontent afin d'obtenir une plus grande domination et contrôle : « l'interprétation de cette lutte est l'objectif central de l'histoire du curriculum » (46). Le même projet est adopté par Ivor Goodson dans ses travaux sur la construction sociale du curriculum, où il analyse le rôle historique de plusieurs groupes (sociaux, économiques, politiques, etc.) dans la définition conflictuelle des disciplines et des plans d'études, démythifiant ainsi l'idée d'un curriculum " atemporel » qui préexisterait aux processus sociaux qui l'ont défini et consolidé (47). Ce même auteur se

(44) Herbert Kliebard : «Constructing a History of the American Curriculum » in : Philip W. Jackson (Ed.) : Handbook of Research on Curriculum, New York, 1992, p. 158.

(45) Idem.

(46) Herbert M. Kliebard : Forging the American Curriculum - Essays in Curriculum History and Theory, New York \& London, 1992, p. xv.

(47) Cf. Ivor F. Goodson : School Subjects and Curriculum Change, New York \& London, 1993, $3^{\mathrm{e}}$ édition, 228 p.; Studying Curriculum: cases and methods, New York, 1994, 152 p.; The Making of Curriculum - Collected Essays, London, 1995, $2^{\mathrm{e}}$ édition, 222 p. 
penche aussi sur la formation des disciplines scolaires, thème qui fut à l'origine d'un des travaux pionniers dans ce champ : The Formation of the School Subjects (1987), où Thomas Popkewitz (48) se propose d'interroger la façon dont les disciplines sont envisagées comme quelque chose d'inévitable, c'est-à-dire une sorte d'incontournable historique, tout en montrant qu'elles ne sont plus que des inventions de ceux qui detiennent le pouvoir de les imposer. Finalement, un mot est dû au livre publié par Michael Apple et Linda Christian-Smith, The Politics of the Textbook (1991), sur les manuels scolaires, un artefact fondamental auquel la recherche n'a pas encore prêté l'attention nécessaire : « Les manuels scolaires ne sont pas uniquement des systèmes de délivrance des faits. Ils sont le résultat de luttes et de compromis politiques, économiques et culturels, étant donné qu'ils sont conçus, organisés et produits par des personnes réelles qui ont des intérêts réels. Et ils sont publiés dans des conditions politiques et économiques définies par les marchés, les ressources et le pouvoir » (49).

D'une façon générale, ces travaux montrent que le processus d'édification du curriculum est d'autant plus efficace qu'il devient « invisible », c'est-à-dire qu'il est pris comme quelque chose qui va de soi. Le concept du curriculum en tant que régulation sociale est souvent utilisé, pour bien souligner les positions et les conflits qui le déterminent, d'abord du point de vue de la conception, mais surtout dans le cadre de sa mise en oeuvre concrète. Il s'agit de problématiser le curriculum en tant que texte, au sens large du terme, qui influence les pratiques pédagogiques, mais qui est aussi adapté ou modifié au cours de son application; le curriculum n'est pas pris comme un ensemble cohérent, d'où les écarts entre les intentions, les réalités et les résultats (50).

Un deuxième domaine de réflexion s'organise autour de la tension entre permanences et changements dans les systèmes scolaires. À ce propos, l'ouvrage classique a été publié par David Tyack, en 1974, The One Best System (51), auteur qui a récemment repris cette question. Dans un texte écrit en collaboration avec William Tobin, il cherche à

(48) Thomas S. Popkewitz (Ed.) : The Formation of the School Subjects - The Struggle for Creating an American Institution, New York \& London, 1987, 310 p.

(49) Michael Apple et Linda Christian-Smith (Eds.) : The Politics of the Textbook, New York \& London, 1991, pp. 1-2.

(50) Cf. encore Michael Apple : «The Politics of a National Curriculum » in : Peter Cookson et Barbara Schneider (Eds.) : Transforming Schools, New York \& London, 1995, pp. 345-370; Ivor F. Goodson et J. Marshall Mangan (Eds.) : History, Context, and Qualitative Methods in the Study of Education, Ontario, 1992, 280 p.; Peter S. Hlebowitsh : Radical Curriculum Theory Reconsidered: A Historical Approach, New York, 1993, 136 p.; Michael E. James (Ed.) : Social Reconstruction through Education: The Philosophy, History, and Curricula of a Radical Idea, Norwood, 1995, $180 \mathrm{p}$.

(51) David B. Tyack : The One Best System - A History of American Urban Education, Cambridge, 1974, $354 \mathrm{p}$. 
comprendre la stabilité des formes scolaires et les difficultés rencontrées par les efforts innovateurs. L'échec de plusieurs initiatives réformatrices, qui n'ont duré que pendant des périodes limitées, est attribué à l'élaboration historique d'une grammaire de l'école qui tend à instaurer comme légitimes certaines manières de faire l'éducation et d'organiser les études. Parmi les conclusions, il faut souligner celle qui concerne les croyances des réformateurs scolaires : « Les réformateurs ont cru que leurs innovations allaient changer les écoles, mais, en effet, ce sont les écoles qui ont changé les réformes. À chaque fois, les enseignants ont choisi, de façon sélective, de mettre en oeuvre ou de modifier les réformes $»(52)$.

Dans ce cadre, il est encore opportun de laisser quelques indications brièves à propos d'une série de recherches qui portent sur les processus d'enseignement et d'apprentissage. Le concept d'alchimie curriculaire - la façon dont les connaissances placées dans leurs lieux scientifiques de référence sont transformées en matière d'enseignement et localisées dans l'espace scolaire - a permis de renouveler l'histoire des didactiques et de l'acte d'enseigner (53). Par ailleurs, la compréhension des stratégies qui ont séparé, historiquement, les connaissances de la méthode, la cognition de l'affection et l'intellect du corps ont facilité une autre approche du travail éducatif. Simultanément, et grâce à des apports psychologiques, de nouveaux éclaircissements ont été fournis sur l'acte d'apprendre, en insistant sur la manière dont chacun s'approprie des possibilités scolaires qui sont mis à sa disposition. Un aspect particulier de ces travaux concerne le renouveau des études sur l'alphabétisation : aujourd'hui, la recherche essaie d'éviter une analyse uniquement centrée sur les « signatures » et d'élargir l'enquête aux usages de la lecture (et de l'écriture) et aux rapports entre les lecteurs et les textes (54).

Les ouvrages publiés par cet ensemble hétérogène d'auteurs ont ouvert le travail historique à toute une série de nouvelles perspectives, aussi bien du point de vue des thèmes que des méthodes. Aux sources classiques (lois, règlements, orientations programmatiques,...) se sont ajoutés des matériaux divers, tels que les travaux des élèves,

(52) David Tyack et William Tobin : « The 'Grammar' of Schooling: Why Has it Been so Hard to Change? », American Educational Research Journal, 31 (3), 1994, p. 478.

(53) Cf., par exemple, Ivor Goodson : «Education as a Practical Matter: some issues and concerns », Cambridge Journal of Education, 25 (2), 1995, pp. 137-148; Thomas S. Popkewitz : « Policy, Knowledge, and Power - Some Issues for the Study of Educational Reform » in : Peter W. Cookson et Barbara Schneider (Eds.) : Transforming Schools, New York \& London, 1995, pp. 413-455.

(54) Carl F. Kaestle : «Introduction », History of Education Quarterly, 30 (4), 1990, pp. 487-491. Cf. aussi Harvey Graff : The Labyrinths of Literacy : Reflections on Literacy Past and Present, Pittsburgh, 1995, 350 p.; Carl F. Kaestle : Literacy in the United States: Readers and reading since 1800, New Haven, 1991, 338 p.; Joseph F. Kett : The Pursuit of Knowledge under Difficulties: From Self-Improvement to Adult Education in America, 1750-1990, Stanford, 1994, 582 p.; Colin Lankshear et Peter L. McLaren (Eds.) : Critical Literacy Politics, Praxis, and the Postmodern, Albany, 1993, 444 p. 
les copies des examens, les journaux des enseignants ou les témoignages oraux. Les analyses quantitatives se doublent d'approches qualitatives, qui accordent une plus grande attention à la production, à la réception et à la localisation des textes dans certains espaces-temps. En croisant l'histoire avec le politique et le subjectif, cette tendance historiographique est en train de produire des recherches fort intéressantes.

\section{Des idées aux discours : Vers une sociohistoire} de la connaissance?

Pendant longtemps les idées pédagogiques ont constitué la référence principale de l'enquête historique. L'interminable recherche des origines et des influences a marqué l'effort pour interpréter la pensée des " grands éducateurs » du passé. Aujourd'hui, le regard se tourne vers la construction, diffusion et réception des discours dans le temps. La réflexion ne s'attarde plus dans le dévoilement des idées, mais cherche, surtout, à comprendre comment chaque époque construit ses auteurs et s'approprie de leurs pratiques discursives. La problématique du pouvoir est au centre de ce mode de travail, qui prétend à identifier comment les discours pédagogiques définissent et redéfinissent les subjectivités, les identités collectives et les savoirs. Le recours aux outils conceptuels de la sociologie de la connaissance devient très utile à l'analyse, entre autres, des langages réformateurs et des discours-experts.

L'un des ouvrages qui illustre le mieux cette perspective de réflexion est $A$ Political Sociology of Educational Reform (1991), écrit par Thomas Popkewitz. L'auteur analyse le langage réformateur au $\mathrm{XIX}^{\mathrm{e}}$ et au $\mathrm{XX}^{\mathrm{e}}$ siècles, mettant en lumière les pratiques de régulation sociale auxquelles il a donné origine. Organisant son travail autour de trois concepts-clef - réforme, professionnalisation et science - l'auteur exploite le fonctionnement des rapports entre pouvoir et savoir au niveau de l'enseignement, de la formation des enseignants et de la recherche éducationnelle. Ses problématiques se définissent autour des questions suivantes : les différents sens accordés au mot réforme à travers les temps et les contextes historiques de l'éducation; les liens entre l'écologie de la réforme et les modèles de régulation sociale qui existent à l'école; les critères élaborés par le travail historique afin de savoir quels ont été les changements produits; la définition du changement comme un problème d'épistémologie sociale, c'est-à-dire comme un moyen pour transformer les connaissances sur l'éducation dans une pratique sociale accessible à l'enquête sociologique.

La production et diffusion de discours-expert dans le champ de l'éducation est une autre des questions qui suscite l'intérêt des historiens. Les sciences de l'éducation s'intègrent dans un programme plus vaste d'affirmation universitaire des sciences humaines en tant que théories et idéologies régulatrices de la vie sociale. La pédagogie 
s'exprime plutôt dans l'espace de la formation des maîtres, l'un des lieux où le langage scientifique acquiert une dominante appliquée et où l'État institue des dispositifs de contrôle d'un groupe professionnel dont l'importance augmente à mesure que l'école obligatoire s'étend à l'ensemble des couches sociales. Il faut bien saisir le rôle de la professionnalisation des connaissances scientifiques et de la consolidation d'un discours-expert dans la redéfinition de l'État et des formes de gouvernementalité au début du $\mathrm{XX}^{\mathrm{e}}$ siècle. Le processus de standardisation et de spécialisation de la connaissance va instituer de nouvelles régulations dans le domaine de l'enseignement. À cet égard, deux axes de réflexion se révèlent particulièrement stimulants : l'analyse de l'émergence et de la consolidation d'un discours scientifique en éducation et l'analyse du développement de pratiques discursives concernant la professionnalisation des enseignants.

Il s'agit, dans le premier cas, de comprendre les difficultés que les sciences de l'éducation ont ressenties dans la reconnaissance du monopole du discours légitime sur son objet, étant donné que leur « autorité » est mise en cause par d'autres discours, aussi bien à l'intérieur (enseignants, administrateurs, élèves) qu'à l'extérieur de l'enceinte scolaire (parents, autorités locales, hommes politiques). L'analyse du savoir spécialisé en éducation se définit, ainsi, par rapport à ses lieux d'encadrement institutionnel et d'utilisation professionnelle des connaissances et par rapport à son sens social et à sa pénétration dans la conscience individuelle et collective. À cet égard, il est intéressant d'étudier la façon dont le langage des sciences de l'éducation s'est imposé comme référent conceptuel pour penser la formation et la profession enseignante. Ce langage se réclame d'une légitimité scientifique, qui sert à instituer des rationalités extérieures aux pratiques éducatives et à consolider un pouvoir régulateur des experts sur le travail des enseignants. Il est le fondement d'un processus de localisation/re-localisation des enseignants et de leur identité professionnelle, tout en assurant un nouveau capital symbolique aux spécialistes (55).

Dans le second cas, la question tient au processus de professionnalisation des enseignants, qui est fonction d'un jeu complexe de pouvoirs (et de hiérarchies) qui construisent différentes communautés discursives à l'intérieur de l'éducation. La consolidation d'un discours spécialisé tend à dévaloriser le savoir des enseignants, c'est-à-dire le savoir issu de l'expérience et de la réflexion sur la pratique. Jürgen Herbst démontre comment le discours de la professionnalisation a contribué à une dépréciation des enseignants et à une réduction de leurs compétences. Il tient des propos très critiques, lorsqu'il dénonce l'ensemble des manifestations « scientifiques » qui ont eu lieu aux universités nord-américaines du début du siècle, lesquelles ont mené à une marginalisation des enseignants et à un renforcement du pouvoir des experts (56). David

(55) Cleo H. Cherryholmes : Power and Criticism: Poststructural Investigations in Education, New York, 1988, 224 p.; Thomas S. Popkewitz, op. cit., 1991.

(56) Jürgen Herbst : And Sadly Teach: Teacher Education and Professionalization in American 
Labaree soutient même que les discours sur la professionnalisation des enseignants sont une stratégie des experts, surtout des formateurs d'enseignants et des chercheurs en éducation, pour augmenter leur statut et leur prestige au sein de l'université (57).

D'après ces auteurs, il est important de bien comprendre comment les discours sur l'éducation ont abouti à la délimitation de catégories qui sont essentielles à nos interprétations. L'élève et l'enseignant, par exemple, ne sont plus investis comme des « individus ", mais comme des " catégories » qui incorporent des manières de regarder et d'agir dans le champ éducatif. Ils sont envisagés comme des " populations » qui sont supposées agir selon certaines règles et principes, devant être soumises à des procédures de gestion sociale. C'est pourquoi il devient impossible de regarder les élèves et les enseignants comme des " personnes ", oubliant le processus historique qui les a formés comme " populations ». Les discours fonctionnent, ainsi, comme des éléments de construction et de configuration d'une réalité sociale, ne se limitant pas à servir de reflet ou de description.

Outre les travaux concernant la production et l'utilisation des statistiques en tant qu'outil de gestion et d'organisation, l'un des domaines où cette approche fut portée plus loin concerne l'étude des enfants inadaptés. Une série de recherches historiques a montré la façon dont les différentes pratiques discursives (arriérés, anormaux, inadaptés,...) ont bâti des catégories populationnelles qui ont fait l'objet d'encadrements sociaux et de politiques éducatives spécifiques ( 58 ). Le travail de Barry Franklin, From " Backwardness » to « At-risk » (1994), analyse d'un point de vue historique les problèmes des enfants avec des difficultés d'apprentissage et les contradictions des réformes éducatives. Travaillant sur une documentation riche et variée, il démontre que les efforts des réformateurs américains pour intégrer les enfants avec des difficultés d'apprentissage les ont conduits par des chemins contradictoires : " Le résultat a été la création d'un éventail de programmes spéciaux pour déplacer ces enfants des classes régulières. Les enfants avec des difficultés d'apprentissage ont été vus différemment pendant le cours du $\mathrm{XX}^{\mathrm{e}}$ siècle. Au début du siècle, on les a vu comme arriérés. Vers le milieu du siècle,

Culture, Madison, 1989, 232 p. Cf. aussi Robert A. Levin : Educating Elementary School Teachers: The Struggle for Coherent Visions, 1909-1978, Lanham, 1994, 188 p.

(57) David Labaree : « Power, knowledge, and the rationalization of teaching: a genealogy of the movement to professionalize teaching », Harvard Educational Review, 62 (2), 1992, pp. 123-154. Cf. aussi du même auteur : « Doing Good, Doing Science: The Holmes Group Reports and the Rhetorics of Educational Reform ", Teachers College Record, 93 (4), 1992, pp. 628-640; « A Disabling Vision: Rhetoric and Reality in Tomorrow's Schools of Education », Teachers College Record, 97 (2), 1995, pp. 166-205.

(58) Cf. par exemple Barry M. Franklin : From « Backwardness » to « At-risk» : Childhood Learning Difficulties and the Contradictions of School Reform, Albany, 1994, 208 p.; Margret A. Winzer : The History of Special Education: From Isolation to Integration, Washington, 1993, 464 p.; Roberta Wollons (Ed.) : Children at Risk in America: History, Concepts, and Public Policy, Albany, 1993, 310 p. 
comme des inadaptés. Aujourd'hui, on les désigne comme étant en risque. Nos étiquettes ont changé, mais notre attachement à la ségrégation s'est maintenu » (59).

En termes méthodologiques, le travail de ces auteurs met l'accent sur les textes et la façon dont l'intertextualité des discours et des pratiques construit les univers sociaux et éducatifs : " Dans les discours-pratiques de l'éducation, les textes se rapportent aux manuels scolaires, aux communications scientifiques, aux monographies, aux orientations curriculaires et aux documents d'évaluation, ainsi qu'aux documents de recherche, aux données de l'observation, aux interventions expérimentales, aux analyses statistiques et aux inférences » (60). Beaucoup plus que la plupart des autres tendances, cet ensemble d'auteurs tient compte des débats récents au sein du champ de l'histoire, tout en essayant d'intégrer dans leurs recherches sur l'éducation des préoccupations linguistiques et théoriques dans l'analyse des langages et dans l'écriture des textes.

\section{Des faits aux politiques : Vers une histoire}

\section{sociopolitique?}

L'éducation vit aujourd'hui un temps de grands débats et interrogations. La vague réformatrice des années quatre-vingt ainsi que les politiques de restructuration des années quatre-vingt-dix sont des signes d'un malaise profond et d'un besoin de changement des systèmes d'enseignement. Une fois le projet historique d'une école de masses édifiée sous l'égide de l'État accompli, bien qu'imparfaitement, il faut reconstituer l'ensemble des croyances individuelles et des investissements sociaux dans l'éducation. Certains auteurs se demandent même si l'on n'est pas face au déclin d'un cycle de deux siècles marqué par l'emprise de l'État et à l'émergence de nouvelles formes d'organisation de l'éducation et de la formation.

Dans un ouvrage récent, curieusement intitulé The End of Education, Neil Postman explique comment les anciens « dieux » (métanarrations) de l'éducation sont tombés en désuétude et pourquoi les nouveaux " dieux » de l'utilité économique, de la consommation, de la technologie et du séparatisme sont inadéquats. À travers cinq métaphores, il présente des « dieux » qui peuvent aider les écoles à redécouvrir un sens pour leur travail : le vaisseau spatial Terre (préserver la Terre comme un thème unificateur), l'ange tombé (organiser les apprentissages d'après la conviction que notre connaissance est imparfaite), l'expérience américaine (souligner les succès et les échecs de

(59) Barry Franklin, op. cit., pp. 153-154.

(60) Cleo Cherryholmes, op. cit., p. 8. 
la nation américaine), la loi de la diversité (mettre en contact les élèves avec toutes les cultures dans leurs forces et faiblesses) et les bâtisseurs de la parole (tenir compte de l'importance du langage dans la construction de notre humanité commune) (61).

Cet article n'est point le lieu adéquat à la critique des thèses de Neil Postman. Pour l'instant, il suffit de bien souligner cette ambiance de redéfinition du projet historique de l'école qui crée les conditions propices au retour d'une histoire sociopolitique de l'éducation. Rien que dans les deux dernières années, il est possible d'identifier toute une série d'ouvrages qui s'attachent à repenser les modèles scolaires et les systèmes éducatifs (62). C'est une réflexion historique fortement ancrée dans les problèmes du présent, bien que ces auteurs soient avertis des pièges du « fonctionnalisme » et de l' « utilitarisme », qui entraînent l'illusion de croire que la raison de l'histoire est d'en tirer des « leçons » pour le présent. Il ne s'agit pas d'écrire une sorte d' « histoire d'introduction », dont la justification implicite est que le passé peut servir d'explication au présent, ce qui oblige à conduire l'enquête d'après des critères qui ne sont pas historiques. Bien au contraire, ces chercheurs n'ignorent pas que l'histoire n'est nécessaire que si elle contribue à créer de nouvelles intelligibilités et à marquer des différences entre le passé et le présent. Ce n'est qu'ainsi que la recherche historique peut être utile, c'est-à-dire peut aider à cultiver un scepticisme avisé, à fournir une culture professionnelle aux éducateurs et à élargir le jeu des possibles.

L'ouvrage coordonné par Diane Ravitch et Maris Vinovskis, Learning from the Past: What History Teaches Us About School Reform (1995), illustre bien ce besoin de reconstituer historiquement les débats éducatifs. Le livre commence par constater les plaintes systématiquement portées à l'égard des écoles, ainsi que les efforts réformateurs qui ont essayé d'y répondre. Les différents chapitres analysent un ensemble varié de problématiques (organisation du système éducatif, apprentissages scolaires, équité et égalité des chances, multiculturalisme, décrochage scolaire,...) dans le but de fournir un encadrement historique aux débats actuels sur l'éducation, démontrant que, au cours des temps, il y a eu des changements majeurs dans les institutions scolaires. Leurs arguments sont construits dans un va-et-vient entre la liberté individuelle et la détermination sociale, de façon à saisir la complexité et la diversité des questions éducationnelles. La réflexion

(61) Neil Postman : The End of Education - Redefining the value of school, New York, 1996, 210 p.

(62) Cf. Michael Apple : Cultural politics and education, New York, 1996, 150 p.; Diane Ravitch : National Standards in American Education - A citizen's guide, Washington, 1995, 224 p.; Francis Schrag : Back to Basics - Fundamental Educational Questions Reexamined, San Francisco, 1995, 180 p.; David Tyack et Larry Cuban : Tinkering toward Utopia: A century of public school reform, Cambridge, 1995, 184 p.; Maris A. Vinovskis : Education, Society, and Economic Opportunity: A Historical Perspective on Persistent Issues, New Haven \& London, 1995, 236 p. Cf. aussi Michael Apple (Ed.) : Review of Research in Education, vol. 21, 1995-1996, 332 p.; Jesse Goodman : " Change without Difference: School Restructuring in Historical Perspective », Harvard Educational Review, 65 (1), 1995, pp. 1-29; Andy Hargreaves : Changing Teachers Changing Times, New York, 1994, 272 p.; Bob Lingard, John Knight et Paige Porter (Eds.) : Schooling Reform in Hard Times, London \& Washington, 1993, 312 p. 
politique, au sens large du terme, permet de dépasser quelques-unes des contraintes explicatives de l'histoire sociale (63).

Trois travaux publiés en 1995-1996 permettent d'expliciter comment quelques auteurs de référence ont investi cette perspective de recherche. Dans Tinkering toward Utopia (1995), David Tyack et Larry Cuban étudient un siècle de réformes de l'école publique. L'idée de départ du livre est très intéressante : faire l'inventaire des questions qu'on se pose couramment sur l'école, les transformant par la suite en objets de recherche historique. Ceci permet la mise en forme d'une réflexion politique sur les rapports entre les intentions réformatrices et leurs conséquences au niveau des pratiques et des institutions scolaires. À travers l'analyse d'une diversité de sources, ils avancent l'hypothèse selon laquelle les changements sont intervenus plutôt à l'extérieur qu'à l'intérieur du « noyau dur » de linstruction : " Pendant le dernier siècle, on vérifie une grande continuité dans la structure, les règles et les pratiques qui organisent le travail d'instruction. Ces regularités organisationnelles - la grammaire de l'école - concernent des pratiques familières telles que le rassemblement des élèves par âge, la division des connaissances en disciplines scolaires et l'affectation d'une classe à un seul enseignant. Dans le noyau dur de l'école les changements ont été lents. Les réformes ont eu lieu, essentiellement, à l'extérieur de ce noyau » (64). Sans se laisser enfermer dans la dichotomie rien ne change/tout change tout le temps, ils expliquent comment les processus historiques ont configuré des croyances personnelles, des langages collectifs et des pratiques institutionnelles qui sont mobilisés au moment de la prise de décision politique en éducation.

Le dernier livre de Maris Vinovskis, Education, Society, and Economic Opportunity (1995), s'encadre dans cette même logique de recherche, approchant une série de « questions persistantes » dans les rapports entre l'éducation et d'autres domaines de la société américaine. Le projet de l'auteur est de fournir une perspective historique aux efforts réformateurs, car s'il est vrai que, depuis le milieu du XIX ${ }^{\mathrm{e}}$ siècle, la plupart des américains a accepté le principe selon lequel tous les enfants devaient recevoir une formation scolaire à titre gratuit, il faut reconnaître « que des désaccords se sont prolongés sur l'ampleur de cette éducation, sur la façon de la payer et sur les moyens de contrôler les écoles » (65). Outre les thèmes de la famille et de la prise en charge des petits enfants, des

(63) Diane Ravitch et Maris Vinovskis : Learning from the Past: What History Teaches Us About School Reform, Baltimore \& London, 1995, 386 p.

(64) David Tyack et Larry Cuban, op. cit., p. 9. Cf. aussi deux autres travaux de David Tyack : « School Governance in the United States: Historical Puzzles and Anomalies » in : Jane Hannaway et Martin Carnoy (Eds.) : Decentralization and School Improvement, San Francisco, 1993, pp. 1-32; « Constructing Difference: Reflections on Schooling and Social Diversity », Teachers College Record, 95 (1), 1993, pp. 8-34.

(65) Maris Vinovskis, op. cit., p. 210. Cf. du même auteur : « The Changing Role of the Federal Government in Educational Research and Statistics », History of Education Quarterly, 36 (2), 1996, pp. 111-128. 
pauvres et des immigrants, Maris Vinosvkis élabore des thèses sur la fréquentation scolaire, la mobilité individuelle et sociale et le soutien économique au développement des systèmes éducatifs. Le concept de mobilité est au centre de sa réflexion, ce qui l'amène à reprendre les débats idéologiques avec les « révisionnistes radicaux », en particulier avec Michael Katz (66). Cet ouvrage donne un apport important à l'approche politique de l'éducation, mettant en oeuvre le jeu historique des contraintes et des possibilités à travers une grille de lecture essentiellement économique.

Finalement, il faut mentionner le travail de Michael Apple, Cultural Politics and Education (1996). Construisant son argument à partir d'une critique aux mouvements conservateurs " restaurationnistes ", il révèle l'épaisseur historique des conflits actuels, tout en élaborant un plaidoyer pour l'école publique : « Beaucoup d'entre nous ont des sentiments ambivalents à l'égard de l'école. Toutes les personnes qui se soucient de ce qui est et ce qui n'est pas enseigné, ainsi que de ceux qui ont ou qui n'ont pas les conditions pour affronter cette réalité, entretiennent des rapports contradictoires avec l'institution scolaire. Nous voulons être des critiques rigoureux, tout en gardant notre engagement et nos espoirs dans la possibilité d'édifier une école pertinente pour les personnes et pour les sociétés. S'il y a un rapport amour/haine, c'est bien ici » (67). Le projet intellectuel de Michael Apple, très marqué du point de vue idéologique, montre clairement l'ambivalence des auteurs critiques vis-à-vis les interactions entre culture et pouvoir à l'intérieur du champ scolaire. À ce propos, il est révélateur des perplexités qui traversent l'usage de l'histoire en vue de la compréhension du présent. Et pourtant, l'analyse d'un éventail large de documents, à laquelle l'auteur se livre dans le but d'établir des ponts entre l'imagination littéraire et les mouvements concrets, expose toutes les potentialités d'une pensée historique et éducationnelle qui donne le primat au politique (68).

L'ensemble de ces travaux soulève trois importantes questions méthodologiques. D'abord, les rapports à un temps proche, ce qui oblige l'historien à cohabiter avec sa propre expérience et à créer des effets de distanciation grâce à des processus de connaissance (identifier l'importance de ce qu'il connaît à travers son expérience personnelle), de mé-connaissance (relativiser les savoirs propres et valoriser d'autres

(66) Maris Vinosvkis s'était déjà engagé dans une polémique contre les "révisionnistes radicaux ", notamment à travers la réalisation d'une étude (The origins of public high schools: A reexamination of the Beverly high school controversy, 1985) qui était une réplique de la recherche originale publié par Michael Katz (The irony of early school reform, 1968).

(67) Michael Apple, op. cit., p. xv. Cf. du même auteur : Official Knowledge - Democratic Education in a Conservative Age, New York \& London, 1993, 226 p.; « Texts and Contexts: The State and Gender in Educational Policy », Curriculum Inquiry, 24 (3), 1994, pp. 349-359.

(68) Cf. à ce propos Carlos Alberto Torres : « State and Education Revisited : Why Educational Researchers Should Think Politically About Education » in : Michael Apple (Ed.) : Review of Research in Education, vol. 21, 1995-1996, pp. 255-331. 
regards) et de re-connaissance (construire des discours et des récits qui sont porteurs de nouvelles façons d'envisager l'histoire). Ensuite, les rapports aux communautés de mémoire qui partagent le même espace social, ce qui crée différentes légitimités dans le travail d'interprétation des événements historiques, obligeant l'historien à agir dans un " terrain contesté ». Finalement, les rapports au changement, ce qui rend plus vive la question de la responsabilité sociale de l'historien, tout en introduisant des spécificités propres dans le débat sur l'espace des positions idéologiques.

À ces trois rapports difficiles, on pourrait en ajouter un quatrième, qui concerne le paradoxe des sources, c'est-à-dire l'abondance et l'inaccessibilité de la plupart des matériaux. Néanmoins, et malgré toutes ces contradictions, l'approche sociopolitique inspire actuellement d'importants changements dans l'histoire américaine de l'éducation. Les dangers d'une histoire portée par les inquiétations du présent sont bien évidents; mais il ne faut jamais oublier que le compromis de l'historien est toujours dans le temps et dans l'espace qu'il habite. L'histoire n'est utile que si « elle fournit des outils de libération contre l'autorité intruse, les credos obsolètes et les affirmations de désespoir » (69). La présence du politique aide à reconsidérer le débat entre la volonté individuelle et l'action collective, de telle façon qu'on retrouve la complexité et l'incertitude qui caractérise toute action humaine aux prises avec sa propre histoire.

\section{Du national au local/global : Vers une histoire comparée?}

Le renouveau des approches comparées constitue une espèce de retour de l'histoire de l'éducation aux sources. En effet, la discipline trouve ses origines au XIX ${ }^{\mathrm{e}}$ siècle, dans un temps historique marqué par la consolidation des systèmes nationaux d'enseignement. La « tradition » inventée par l'histoire de l'éducation sert à légitimer les efforts des réformateurs scolaires; du fait, il n'est pas étonnant de constater qu'à cette époque beaucoup d'hommes politiques se sont adonnés à l'histoire (et vice-versa). La notion d'identité nationale est constitutive des projets éducatifs qui essaient de façonner les citoyens des « nouveaux » États-nation. L'histoire de l'éducation est censée établir les limites, c'est-à-dire les différences et les similitudes, ce qui implique une réflexion comparée, bien présente, vers la fin du XIX ${ }^{\mathrm{e}}$ siècle, dans les manuels de la discipline et dans les préambules des lois de réforme.

Aujourd'hui, le principe d'identité nationale ne fonctionne plus comme élément référentiel des politiques éducatives, ce qui entraîne une présence plus forte des

(69) Joyce Appleby, Lynn Hunr et Margaret Jacob : Telling the Truth about History, New York \& London, 1994, p. 308. 
régulations locales et globales. Des auteurs comme Ernest Gellner, Anthony Smith ou Charles Tilly (70) attirent l'attention sur les liens entre la modernité en tant que structure de pensée et la nation en tant que système idéologique, mettant en cause l'idée de la nation comme forme « naturelle » d'organisation des peuples. Benedict Anderson va même plus loin, considérant que la nation est une communauté politique imaginée : " En réalité, toutes les communautés plus larges que les petits villages où il y a un contact face à face (et encore!) sont imaginées. Les communautés se différencient non pas par leur fausseté/authenticité, mais par la façon à travers laquelle elles sont imaginées » (71). Simultanément, il y a des mouvements de reprise de la nation en tant que principe identitaire et concept organisationnel, et on ne doit pas ignorer que les processus de globalisation tendent, souvent, à renforcer les tendances nationalistes.

Il faut toutefois reconnaître qu'on est confronté à une véritable explosion des identités traditionnelles et à un effort de redécouverte, ou plutôt de réinvention, de nouvelles identités. Ce ne sont pas nécessairement des identités territorialisées, car les communautés de sens prennent de plus en plus le relais des communautés localisées dans la consolidation des liens d'attachement et de solidarité. La conclusion de l'ouvrage de Yasemin Soysal sur les limites de la citoyenneté est en elle-même un défi aux systèmes éducatifs tels qu'ils se sont développés tout le long des deux derniers siècles: «La citoyenneté nationale, la tradition inventée du XIX ${ }^{\mathrm{e}}$ siècle, et les sentiments habituels dont elle est porteuse resteront avec nous pendant un certain temps. Néanmoins, il faut reconnaître que la citoyenneté nationale n'est plus un concept adéquat pour soutenir une narrative raisonnée du statut d'adhésion dans la période de l'après-guerre. Les formations post-nationales nous invitent à repenser nos définitions et conceptions théoriques sur la citoyenneté et l'État-nation » (72).

C'est à l'intérieur de cette interrogation fondamentale sur l'idée de nation et sur le rôle de l'école dans l'affirmation d'une citoyenneté nationale que se développent les perspectives historiographiques prônant un retour au comparatisme. L'enjeu d'une histoire comparée de l'éducation est celui d'élargir l'enquête vers l'infiniment grand des processus de globalisation et l'infiniment petit des phénomènes de localisation, mettant sur pied des encadrements conceptuels capables d'aller au-delà de l'étude inter-nationale des systèmes éducatifs. Pour cela, il faut se doter des outils théoriques et méthodologiques qui

(70) Cf. Ernest Gellner : Encounters with Nationalism, Oxford, 1994, 208 p.; Anthony Smith : National Identity, Reno, 1991, 228 p.; Charles Tilly : Coercion, Capital, and European States, AD 990-1992, Cambridge, 1992, 272 p.

(71) Benedict Anderson : Imagined Communities - Reflections on the Origin and Spread of Nationalism, New York \& London, 1991, $2^{\mathrm{e}}$ édition, p. 6.

(72) Yasemin N. Soysal : Limits of Citizenship - Migrants and Postnational Membership in Europe, Chicago \& London, 1994, p. 167. 
permettent de saisir l'historicité des faits éducatifs, qui ne sont pas des réalités naturelles, mais des constructions sociales imprégnées de passé. La liaison entre l'histoire et la comparaison n'est pas facile, mais elle est porteuse de nouveaux enjeux de connaissance dans l'arène de l'éducation.

L'une des tendances les plus fructueuses - malgré les critiques fondées des historiens qui réagissent à des interprétations qui ne prennent pas suffisamment en compte les contextes nationaux - est représentée par les auteurs qui travaillent selon l'approche du système mondial. Dans leurs ouvrages, notamment sur l'école de masses et le développement du curriculum, ils montrent bien le caractère transnational de phénomènes qu'on s'était habitué à lire uniquement sur le plan national. En ce qui concerne l'école de masses, par exemple, ils suggèrent qu'on est face à une institution mondiale, aussi bien en tant que principe normatif qu'en tant que réalité organisationnelle. Les conclusions d'une étude menée sur 120 pays, concernant la période 1870-1980, sont assez éloquentes : « Le développement de l'école de masses dans un pays donné est peu influencé par des caractéristiques nationales, telles que l'urbanisation, la composition raciale ou religieuse, l'indépendance ou même la prescription légale de la scolarité obligatoire. Le développement de l'école de masses est fortement influencé par la localisation structurelle du pays dans le système mondial » (73).

Un autre exemple des travaux conduits selon cette approche concerne les résultats d'une enquête sur le développement du curriculum au niveau mondial depuis de début du $\mathrm{XX}^{\mathrm{e}}$ siècle. Travaillant sur des données recueillies dans plus d'une centaine de pays, les auteurs constatent une relative homogénéité dans l'organisation du curriculum, tout en soulignant que la variation entre les sociétés nationales est moins significative qu'on ne l'aurait attendu : « La société qui construit le curriculum de l'école de masses est un modèle imaginé beaucoup plus qu'une réalité immédiate; est mondiale plutôt que locale ou nationale; et possède des dispositifs organisationnels et professionnels qui sont une conséquence de cette mondialisation. (...) Une des principales surprises de notre recherche porte, non sur l'insignifiance du local, mais plutôt sur l'insignifiance du national, dans l'explication des logiques de développement du curriculum de l'école de masses » (74).

(73) John W. Meyer, Francisco Ramirez et Yasemin Soysal : « World Expansion of Mass Education, 1870-1980 », Sociology of Education, 65 (2), 1992, p. 146. Cf. aussi John Boli et Francisco Ramirez : « Compulsory Schooling in the Western Cultural Context » in : Robert Arnove, Philipe Altbach et Gail Kelly (Eds.) : Emergent Issues in Education: Comparative Perspectives, Albany, 1992, pp. 25-38; Francisco O. Ramirez et Marc J. Ventresca : « Building the Institution of Mass Schooling: Isomorphism in the Modern World » in : Bruce Fuller et Richard Rubinson (Eds.) : The Political Construction of Education - The State, School Expansion, and Economic Change, New York, 1992, pp. 47-59; Yasemin N. Soysal et David Strang : « Construction of the First Mass Education Systems in Nineteenth-century Europe », Sociology of Education, 62, 1989, pp. 277-288.

(74) John W. Meyer, David H. Kamens et Aaron Benavot : School Knowledge for the Masses: World Models and National Primary Curricular Categories in the Twentieth Century, London, 1992, pp. 6-7. 
L'idée que l'éducation n'est pas une institution nationale, mais plutôt une composante rationalisée d'une technologie mondiale de progrès et de modernisation, est lourde de conséquences pour la réflexion historique et comparée. C'est une idée qu'il faut mettre en rapport avec le processus de globalisation, même si ce concept soulève d'importantes résistances théoriques, étant donné qu'il tend à reproduire la dichotomie centre-périphérie et à imposer des principes d'explication qui suffoquent d'autres niveaux d'analyse et de compréhension de la réalité. Mais, si au lieu de tenir le discours d'une " culture globale ", on cherche à comprendre comment les évolutions mondiales sont appropriées par les différentes communautés, cette approche contient trois grands avantages : la possibilité d'une mise en perspective historique, l'adoption d'un regard qui n'est pas eurocentrique et la maîtrise d'un objet théoriquement bien défini (75). C'est une perspective qui s'avère très stimulante pour l'analyse d'une série de thèmes éducatifs (le développement du curriculum, la scolarité obligatoire, les taux de scolarisation, l'expansion scolaire,...), pourvu qu'on soit capable de contrôler une explication surdeterminée par le " global » et d'installer des dispositifs méthodologiques faisant un va-et-vient entre le macro (banques de données au niveau international) et le micro (études de cas) (76).

Un deuxième courant de recherche essaie de valoriser les aspects linguistiques, adoptant quelques-unes des idées-clef de la " rupture postmoderne ». Le projet scientifique dont ces auteurs sont porteurs est assez clair : passer de l'analyse des « faits » à l'analyse du « sens historique des faits ». D'où l'émergence d'une nouvelle épistémologie de la connaissance, qui définit des perspectives de travail centrées non seulement sur la matérialité des faits éducatifs, mais aussi sur les communautés discursives qui les décrivent, les interprètent et les localisent dans un espace-temps donné. La dimension historique contribue à clarifier et à articuler conceptuellement la comparaison : les phénomènes éducatifs, et les pratiques discursives qui leur accordent du sens, sont vus comme un savoir particulier, historiquement formé, qui légitime des manières d'agir, de sentir, de parler et de voir le monde (77).

(75) Cf. Christel Adick : « Education in the Modern World System : An Attempt to End the Mythology of the Concept of Education as a Colonial Heritage », Education, 40, 1989, pp. 30-48.

(76) Cf. encore Robert F. Arnove, Philip G. Altbach et Gail P. Kelly (Eds.) : Emergent Issues in Education - Comparative Perspectives, Albany, 1992, 364 p.; Bruce Fuller et Richard Rubinson (Eds.) : The Political Construction of Education - The State, School Expansion, and Economic Change, New York, 1992, 262 p.; Mark B. Ginsburg (Ed.) : Understanding Educational Reform in Global Context - Economy, Ideology, and the State, New York \& London, 1991, 404 p.

(77) Cf. Rolland G. Paulston (Ed.) : Social Cartography - Mapping Ways of Seeing Social and Educational Change, New York \& London, 1996, 458 p.; Thomas S. Popkewitz (Ed.) : Changing Patterns of Power - Social Regulation and Teacher Education Reform, Albany, 1993, 382 p.; Val D. Rust : " Postmodernism and Its Comparative Education Implications », Comparative Education Review, 35 (4), 1991, pp. 610-626. 
Du point de vue théorique, ces auteurs cherchent à mettre sur pied de nouvelles intelligibilités, sur la base d'une reconciliation entre l'histoire et la comparaison. L'analyse ne prend plus comme référence des contextes définis selon la visibilité de leurs contours géographiques, politiques ou sociaux, mais des contextes définis selon l'invisibilité des pratiques discursives qui leur donnent sens. Il y a un effort pour dépasser un regard centré sur les niveaux intermédiaires de décision et pour saisir toute la complexité des phénomènes de globalisation et de localisation, envisagés comme deux moments d'un même processus historique. Portés par le projet de considérer le monde comme un texte, ces auteurs veulent comprendre comment les discours font partie des pouvoirs qui partagent et divisent les hommes et les sociétés, qui entérinent des situations de dépendance et des logiques de discrimination, qui construisent des manières de penser et d'agir. Les questions de l'identité sont au coeur de leurs soucis, ainsi que des thématiques telles que la construction du curriculum, la formation des disciplines scolaires, les nouvelles régulations politiques et sociales, les rapports au pouvoir ou la consolidation des formes « légitimes » de connaissance scolaire.

Aujourd'hui, le débat méthodologique est très intense au sein des cercles de la recherche comparée (78). Il y a une conscience forte des limites des interprétations et des fragilités des encadrements théoriques et conceptuels. L'histoire comparée de l'éducation a encore un long chemin à parcourir, notamment en ce qui concerne la production des outils méthodologiques les plus pertinents. Mais il est impossible de passer outre aux potentialités de cette ligne de réflexion. Dans un monde qui, malgré la recrudescence des nationalismes, ne s'imagine plus uniquement à l'intérieur des frontières nationales, il est utile de penser une histoire qui se projette dans une pluralité d'espaces et de lieux d'attachement.

En tant qu'espace de référence des processus identitaires, l'analyse comparée de l'éducation exige qu'on consacre plus d'attention à l'histoire et à la théorie au détriment d'une pure description, aux contenus de l'éducation et pas seulement à ses résultats, aux méthodes qualitatives et ethnographiques au lieu d'un recours exclusif à la quantification et aux données statistiques. En tant qu'élément central des logiques de globalisation économique et culturelle, l'éducation constitue un terrain particulièrement stimulant pour un travail comparatif capable de faire ressortir l'épaisseur historique des débats pédagogiques et des projets scolaires. Mais pour cela il faut eviter une espèce de réflexion molle du point de vue conceptuel et produire une pensée critique capable d'assumer sa propre historicité et de s'investir dans l'élaboration d'approches compréhensives.

(78) Cf. Robin J. Burns et Anthony R. Welch (Eds.) : Contemporary perspectives in comparative education, New York, 1992, 432 p.; R. Murray Thomas (Ed.) : International comparative education: practices, issues and prospects, Oxford \& New York, 1990, 338 p.; Anthony R. Welch : « Class, Culture and the State in Comparative Education: problems, perspectives and prospects », Comparative Education, 29 (1), 1993, p. 23. 
La première et la dernière des tendances présentées renvoient à des approches qui ont des contours spécifiques aux États-Unis. Il s'agit, d'une part, de l'histoire politique et culturelle des « sans-voix » (minorités, femmes,...) et, d'autre part, des analyses organisées sur la base du concept de "globalisation ». C'est vrai que la recherche européenne n'est pas insensible à ces thèmes, mais la façon dont ils se sont historiquement constitués en objets scientifiques établit des différences assez nettes entre les historiographies des deux continents. En outre, on ne doit pas oublier que, dans l'espace américain, ces tendances se sont développées en liaison étroite avec des projets d'action politique, aussi bien en ce qui concerne les questions de l'identité et de la participation à la vie sociale, que par rapport à une vision impérialiste qui se traduit par la consécration de catégories de pensée qui deviennent incontournables.

Pour ce qui est des autres tendances, il est assez facile d'identifier des ressemblances et de bâtir des comparaisons. Il suffit de compulser quelques-unes des revues publiées en Europe (Histoire de l'Éducation, Historia de la Educación, History of Education, Paedagogica Historica,...) pour confirmer la proximité des objets d'étude, des encadrements conceptuels et des outils méthodologiques. La constatation n'est pas surprenante, étant donné la circulation des idées et le brassage intellectuel au niveau international, mais aussi l'existence de problématiques communes qui induisent des réponses semblables.

Néanmoins, il est possible de désigner quelques dispositions particulières de la recherche américaine, qui tiennent surtout à l'histoire du pays et au mode de fonctionnement de la communauté scientifique. Signalons, d'abord, l'attention privilégiée à l'histoire du $\mathrm{XIX}^{\mathrm{e}}$ et du $\mathrm{XX}^{\mathrm{e}}$ siècles, ce qui établit un rapport aux sources très différent de celui qu'on connaît en Europe, tout en suggérant une explication historique portée par le présent. Mentionnons, ensuite, une plus grande fluidité entre les disciplines scientifiques, ainsi qu'une plus grande préoccupation avec les questions théoriques et linguistiques. Soulignons, en troisième lieu, la grande mobilité institutionnelle et géographique des chercheurs, ce qui entraîne une moindre fixation sur certains objets de travail, notamment quant à leurs limites territoriales. Évoquons, finalement, une sensibilité bien définie en ce qui concerne les dimensions comparatives, ce qui tient au rôle que la communauté 
scientifique américaine exerce un peu partout dans le monde.

À ces quatre aspects, on pourrait ajouter la mise sur pied par les chercheurs de stratégies propres de reconnaissance, aussi bien parmi leurs pairs, qu'à l'extérieur du groupe disciplinaire; la diffusion auprès de scientifiques d'autres disciplines est même le signe d'un prestige solide et reconnu. Les références antérieures révèlent des manières de travailler qui favorisent le passage de la réflexion historique à la pensée éducationnelle (et vice-versa); il est assez courant que les historiens américains écrivent aussi sur des thèmes d'actualité, prenant partie dans les débats éducationnels et pédagogiques de nos jours.

Ceci marque des manières d'être et des attitudes vis-à-vis le travail intellectuel qui sont porteuses de nouvelles matrices d'explication historique. Parmi leurs enjeux le moindre n'est pas celui de montrer l'historicité profonde des problèmes de l'éducation et l'impossibilité de saisir les dilemmes pédagogiques sans comprendre comment ils se sont formés historiquement. Est-ce un enjeu différent du nôtre? 